\title{
Transcriptome analysis of atemoya pericarp elucidates the role of polysaccharide metabolism in fruit ripening and cracking after harvest
}

Jingjing Chen ${ }^{1,2}$, Yajie Duan ${ }^{1,2}$, Yulin Hu ${ }^{1,2}$, Weiming $\mathrm{Li}^{1,2}$, Dequan Sun ${ }^{1,2}$, Huigang $\mathrm{Hu}^{1,2^{*}}$ and Jianghui Xie ${ }^{1,2^{*}}$

\begin{abstract}
Background: Mature fruit cracking during the normal season in African Pride (AP) atemoya is a major problem in postharvest storage. Our current understanding of the molecular mechanism underlying fruit cracking is limited. The aim of this study was to unravel the role starch degradation and cell wall polysaccharide metabolism in fruit ripening and cracking after harvest through transcriptome analysis.

Results: Transcriptome analysis of AP atemoya pericarp from cracking fruits of ethylene treatments and controls was performed. KEGG pathway analysis revealed that the starch and sucrose metabolism pathway was significantly enriched, and approximately 39 DEGs could be functionally annotated, which included starch, cellulose, pectin, and other sugar metabolism-related genes. Starch, protopectin, and soluble pectin contents among the different cracking stages after ethylene treatment and the controls were monitored. The results revealed that ethylene accelerated starch degradation, inhibited protopectin synthesis, and enhanced the soluble pectin content, compared to the control, which coincides with the phenotype of ethylene-induced fruit cracking. Key genes implicated in the starch, pectin, and cellulose degradation were further investigated using RT-qPCR analysis. The results revealed that alpha-amylase 1 (AMY1), alpha-amylase 3 (AMY3), beta-amylase 1 (BAM1), beta-amylase 3 (BAM3), beta-amylase 9 (BAM9), pullulanase (PUL), and glycogen debranching enzyme (glgX), were the major genes involved in starch degradation. AMY1, BAM3, $B A M 9, P U L$, and glgX all were upregulated and had higher expression levels with ethylene treatment compared to the controls, suggesting that ethylene treatment may be responsible for accelerating starch degradation. The expression profile of alpha-1,4-galacturonosyltransferase (GAUT) and granule-bound starch synthase (GBSS) coincided with protopectin content changes and could involve protopectin synthesis. Pectinesterase (PE), polygalacturonase $(P G)$, and pectate lyase (PEL) all involved in pectin degradation; PE was significantly upregulated by ethylene and was the key enzyme implicated pectin degradation.

Conclusion: Both KEGG pathway enrichment analysis of DEGs and material content analysis confirmed that starch decomposition into soluble sugars and cell wall polysaccharides metabolism are closely related to the ripening and cracking of AP atemoya. A link between gene up- or downregulation during different cracking stages of atemoya fruits and how their expression affects starch and pectin contents were established by RT-qPCR analysis.
\end{abstract}

\footnotetext{
* Correspondence: huhuigang@sina.com; 61984011@qq.com

${ }^{1}$ Key Laboratory of Tropical Fruit Biology, Ministry of Agriculture, South

Subtropical Crops Research Institute, Chinese Academy of Tropical

Agricultural Sciences, Zhanjiang 524091, China

Full list of author information is available at the end of the article
}

(c) The Author(s). 2019 Open Access This article is distributed under the terms of the Creative Commons Attribution 4.0 International License (http://creativecommons.org/licenses/by/4.0/), which permits unrestricted use, distribution, and reproduction in any medium, provided you give appropriate credit to the original author(s) and the source, provide a link to the Creative Commons license, and indicate if changes were made. The Creative Commons Public Domain Dedication waiver (http://creativecommons.org/publicdomain/zero/1.0/) applies to the data made available in this article, unless otherwise stated. 


\section{Background}

African Pride (AP) belongs to atemoya (Annona cherimola Mill $\times$ A. squamosa L.), a semi-deciduous, exotic subtropical fruit that is consumed in various countries [1]. It readily softens and cracks, thus reducing marketable yield and quality, and in turn promotes disease [2]. Fruit cracking during atemoya ripening initially develops around the peduncle and then radiates outward from the fruit base. It can occur before and after harvest, and the amount varies with cultivar. A wide range of stimuli that varies among species triggers fruit cracking. Correlations have been observed between susceptibility of fruit cracking and specific fruit traits, such as shape [3], size [4], firmness [5], stomata in fruit skin [6], cuticular properties [7], osmotic concentration, and other factors [8]. Fruit cracking in AP atemoya more commonly occurs during postharvest storage. Some measures have been adopted to delay cracking, including low temperature storage, 1-methylcyclopropene (1-MCP) treatment [9], chitosan and citric acid treatment [10], and salicylic acid treatment [11]. However, these could not effectively prevent fruit cracking, and our current understanding of the molecular mechanism underlying fruit cracking is limited.

Atemoya is a fruit with typical climacteric maturation, and studies have shown that fruit cracking starts at the respiratory peak and as ethylene production increases [2, 12-14]. Generally, atemoya softening is accompanied by cracking. Sugar metabolism is the most important material transformation process in atemoya fruit ripening, including starch degradation, transformation, and cell polysaccharides metabolism. It has been reported that fruit softening is typically accompanied by the depolymerization and solubilization of various classes of cell wall polysaccharides, such as pectins and hemicelluloses, and by elevated levels of the expression of genes, proteins, and enzyme activities associated with cell wall degradation $[15,16]$. However, our current understanding of the molecular mechanism underlying fruit cracking is limited.

Only a few studies on the elucidation of the molecular mechanism of atemoya cracking have been conducted to date. Several cell wall-related genes, cell wall relaxation factors, and cuticle-related genes have been identified, including polygalacturonase $(P G)$, pectinesterase $(P E), \beta$-galactosidase $(\beta-G a l)$, expansins $(E X P)$, xyloglucan endotransglycosylase (XET), AP2/EREBP-type transcription factor (PaWINB), wax synthase (WS), and $\beta$-ketoacyl-CoA synthase (PaKCS6) [8, 17-23], are associated with fruit softening and cracking. Whole genome sequencing of atemoya has not been performed to date, and only a few genetic resources are currently available. The objective of this study was to identify cracking-related metabolic pathways and genes in atemoya fruits after ethylene treatment and in controls during postharvest through transcriptome sequencing and to establish a solid foundation for future molecular studies using high-throughput sequencing and expression data analysis.

\section{Results}

Appearance, firmness, and TSS changes in atemoya fruits after ethylene treatment and in controls

AP summer fruits are generally ready to eat about 3 or 4 days after fruit harvest ( $80 \%$ ripening). When the skin color changes from dark green to light green [14, 24], the surface of the fruit is cream in color between segments, and the surface of the separate fruit carpels becomes smooth [2], the fruits are harvested. However, in addition to the above two conditions, the definition of $80 \%$ ripening also includes a $10 \%$ soluble solids content [24]. Under room temperature and in the summer in Zhanjiang City, China, fruits started to separate between the peduncle and central axis 2 days after harvest as shown as NT-PC-1 in Fig. 1a. We used penetration tests to evaluate atemoya softening and found that the firmness value of fruits was too high to determine, but TSS levels at day $2(11.6 \%)$ were higher than those on day $0 /$ NT-0 (9.5\%). On day 3, cracking radiated out from the fruit base, as shown as NT-PC-2 in Fig. 1a, and the fruits started to soften, allowing us to measure firmness. The TSS levels continued to increase to $21 \%$. On day 4 , cracking was deeper, radiating from the peduncle to the fruit top in some fruits, thereby completely exposing the peduncle as shown as NT-PC-3 in Fig. 1a. The firmness of the fruits rapidly decreased, and the highest TSS values $(25 \%)$ were observed.

After $24 \mathrm{~h}$ of ethylene treatment (Eth-24 h), no difference in appearance between atemoya fruits on day $0 /$ NT-0 was observed, although TSS content was slightly higher than that on day 0/NT-0. On day 2, the peel started to show signs of cracking, and regions around the peel had softened. On the third day, the fruits showed more extensive and deeper cracking, including the peduncle as shown as Eth-PC in Fig. 1a. Fruit firmness rapidly decreased and the highest TSS values were observed (Fig. 1b). However, some fruits subjected to ethylene treatment did not show signs of cracking, but exhibited similar changes in firmness and TSS content as that in cracking fruits shown as Eth-NC in Fig. 1.

\section{Transcriptome sequencing, assembly, and annotation}

To obtain a reference transcriptome for cracking atemoya fruits, a RNA-Seq library was constructed using RNAs from the pericarp, and seven libraries were generated from untreated fruits at room temperature (NT-0, NT-PC-1, NT-PC-2, and NT-PC-3) and ethylene-treated fruits (Eth-24h, Eth-PC, and Eth-NC) at different cracking phases. These libraries were sequenced using the Illumina Hiseq 2500 platform. Table 1 shows that we 

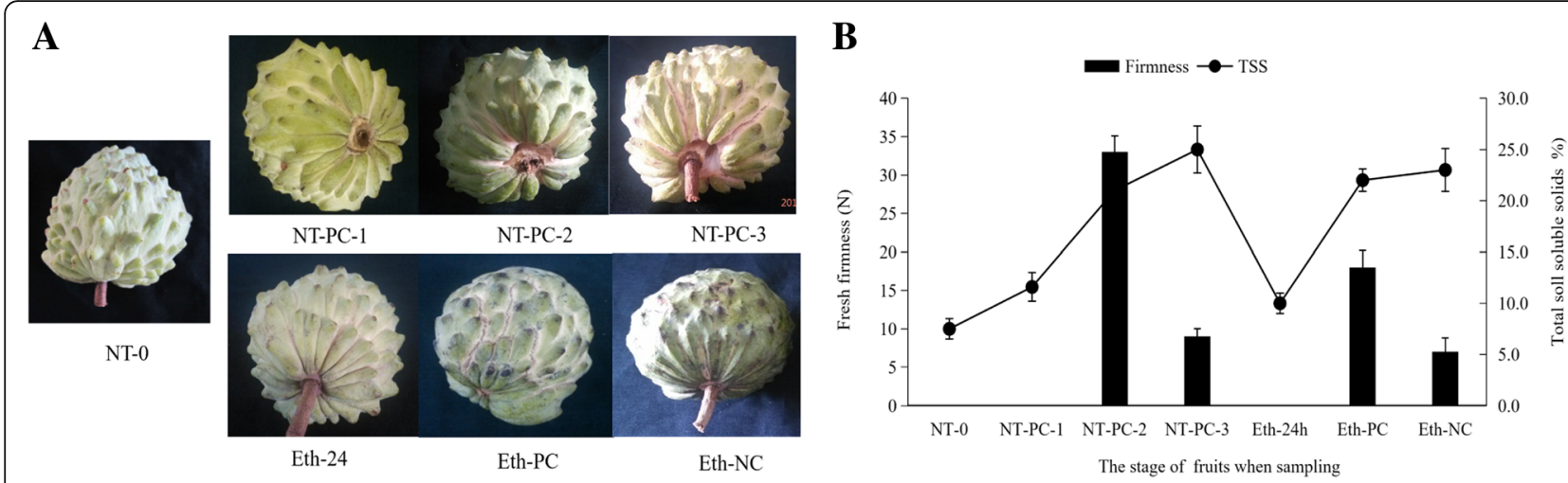

Fig. 1 Appearance, firmness, and TSS content of atemoya fruits at postharvest. a Atemoya fruits during postharvest storage with or without ethylene treatment. Samples corresponding to various cracking degrees designated as NT-0, NT-PC-1, NT-PC-2, and NT-PC-3 for normal treatment and Eth-24 h, Eth-PC, and Eth-NC for the ethylene treatment were selected for transcriptome sequencing. $\mathbf{b}$ Firmness values and TSS contents after ethylene and no treatments. Vertical bars represent the standard error of three biological replicates

generated a total of $38 \mathrm{~Gb}$ of nucleotides with a Q30 percentage of $95 \%$, and the percentage of unassigned "N" bases was nearly 0 . The distribution of reads in the seven libraries was 50.9 million (13.34\%) for the control (NT-0), 57.1 million (14.97\%) for NT-PC-1, 49.4 million (12.95\%) for NT-PC-2, 50.0 (13.10\%) for NT-PC-3, and 69.58 million (14.97\%) for Eth-24h, 47.4 (18.23\%) for Eth-PC, and 38.2 (12.42\%) for Eth-NC. After cleaning and performing quality checks, the Trinity package was used to performed de novo assembly of 64,069 unigenes, with a mean size of $670 \mathrm{bp}$. The size distributions of these unigenes are shown in Additional file 1. After searching for homologous sequences using BLASTX against Nr, UniProt, GO, KEGG, and COG databases, a total of $39.14 \%(25,074 / 64,069)$ unigenes could be assigned to a homolog in all five databases (Fig. 2a). About $38.97 \%(24,967 / 64,069)$ of the unigenes could be annotated by BLASTx (E-value <1e-5) using the NCBI $\mathrm{Nr}$ database. Based on the E-value distribution, we found that approximately $42.56 \%$ of the unigenes showed very strong homology (E-value $<1 \mathrm{e}-100)$ to available plant sequences (Fig. 2b). Figure 2c shows that approximately 12,705 unigenes were annotated to five top-hit species, including Nelumbo nucifera, Vitis vinifera, Elaeis guineensis, and Glycine max.

Approximately 17,039 unigenes could be classified into three GO categories, namely, cellular component, biological process, and molecular function (Additional file 2). In addition, 12,650 and 5780 unigenes could be annotated according to UniProt and COG, respectively (Additional file 3). Around 4932 unigenes in the atemoya pericarp were mapped to 203 KEGG pathways (Additional file 4). The maps with the highest unigenes representation included metabolic pathways (map01100) and ribosome pathway (ko03010), followed by microbial metabolism in diverse environments (map01120), ribosome (map03010), plant hormone signal transduction (map04075), plant-pathogen interaction (map04626), spliceosome (map03040), RNA transport (map03013), protein processing in endoplasmic reticulum (map04141), ubiquitin-mediated proteolysis (map04120), and starch and sucrose metabolism (map00500) (Additional file 4).

Table 1 Throughput and quality of RNA-seq of the reference library and the DGE libraries

\begin{tabular}{|c|c|c|c|c|c|c|}
\hline Libraries & Clean Reads Number & Clean Reads Rate (\%) & Clean Bases Number & Ns Reads Number & Ns Reads Rate (\%) & Clean Q30 Bases Rate (\%) \\
\hline$\overline{N T-0}$ & $50,896,590$ & 85.60 & $5,089,659,000$ & 1104 & 0 & 95.68 \\
\hline NT-PC-1 & $57,121,252$ & 86.41 & $5,712,125,200$ & 1522 & 0 & 95.08 \\
\hline NT-PC-2 & $49,432,496$ & 87.13 & $4,943,249,600$ & 1382 & 0 & 95.33 \\
\hline NT-PC-3 & $50,004,384$ & 87.49 & $5,000,438,400$ & 1488 & 0 & 95.38 \\
\hline Eth-24 h & $57,160,978$ & 86.81 & $5,716,097,800$ & 1620 & 0 & 95.22 \\
\hline Eth-PC & $69,577,816$ & 87.70 & $6,957,781,600$ & 1910 & 0 & 95.34 \\
\hline Eth-NC & $47,402,348$ & 85.38 & $4,740,234,800$ & 1294 & 0 & 95.16 \\
\hline Total & $381,595,864$ & & $38,159,586,400$ & & & \\
\hline
\end{tabular}




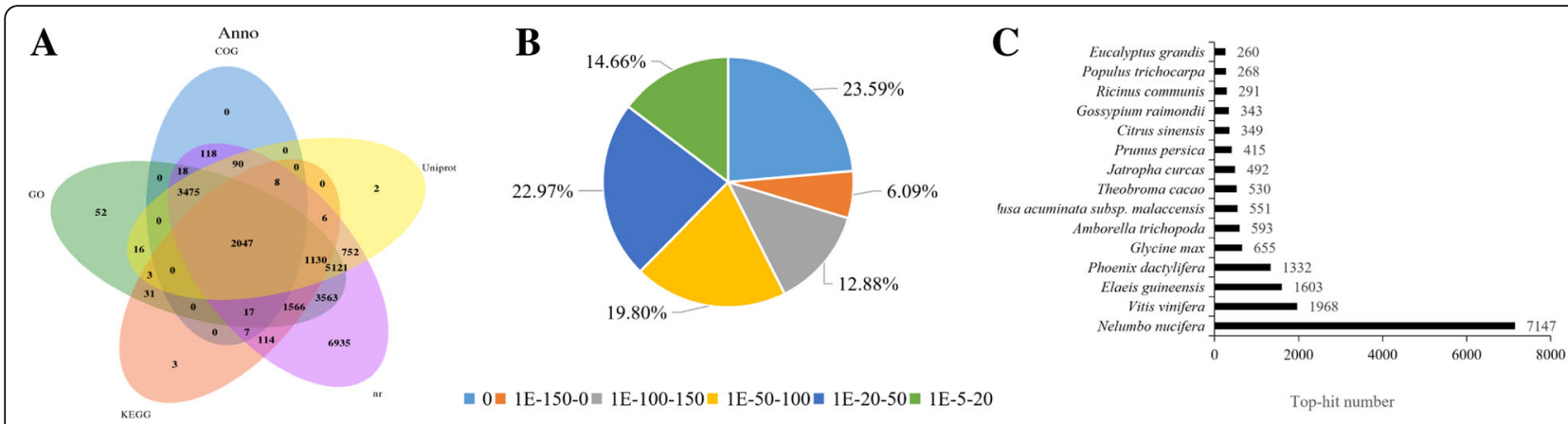

Fig. 2 Characteristics of homology search of atemoya unigenes. a Venn diagram of number of unigenes annotated by BLASTx with a cut-off E-value 1e- 05 against protein databases. Numbers in the circles indicate the number of unigenes annotated by single or multiple databases. $\mathbf{b}$ E-value distribution of the top BLASTx hits against the Nr database. $\mathbf{c}$ Number of unigenes matching the 25 top species using BLASTx in the $\mathrm{Nr}$ database

\section{Screening and KEGG pathway enrichment analysis of differentially expressed genes (DEGs)}

We measured the unigene expression levels based on the obtained unique reads from seven libraries using RPKM values (Additional file 5), and then screened out DEGs using the combined criteria of FDR $\leq 0.05$ and the $\mid \log 2$ ratio $\mid \geq 1$. The library generated from the pooled NT-0 samples was used to normalize the DEGs in the other six libraries, which was then followed by pairwise comparisons. Changes in gene expression were determined by comparing NT-PC-1/NT-0, NT-PC-2/NT-0, NT-PC-3/NT-0, Eth-24h/NT-0, Eth-PC/NT-0, and Eth-NC/NT-0, respectively. NT-PC-1/NT-0, NT-PC-2/ NT-0, and NT-PC-3/NT-0 were defined as the first group, and Eth-24 h/NT-0, Eth-PC/NT-0, and Eth-NC/ NT-0 were designated as the second group. In these pairwise comparisons, we detected both unique and overlapping sets of DEGs. Figure 3a shows an enrichment of downregulated genes compared to upregulated genes during fruit softening and cracking. A total of $22,305,30,072$, and 35,176 DEGs were detected by analyzing NT-PC-1/NT-0, NT-PC-2/NT-0, and NT-PC-3/ NT-0, respectively. A total of 42,513 DEGs, including 15,767 overlapping genes, were enriched during normal postharvest storage. For ethylene treatment, Eth-24 h/ NT-0, Eth-PC/NT-0, and Eth-NC/NT-0, 49,497 DEGs showed enrichment, which included 11,811 overlapping genes. By comparing the first group and the second group, a total of 54,819 DEGs, including 5322 unique genes in the first group, 12,306 unique genes in the second group, and 37,191 overlapping genes were obtained (Fig. 3b).

Four libraries, including NT-0, NT-PC-1, NT-PC-2, and NT-PC-3, were selected and used in DEG profiling, and these libraries did not undergo treatment and exhibited cracking during fruit ripening. The number of up-and downregulated DEGs was determined by paired comparison of the four libraries, which was then followed by KEGG pathway enrichment analysis. The top 20 KEGG pathways with the highest number of upand downregulated DEGs are shown in Fig. 4. Starch and sucrose metabolism (ko00500), plant hormone signal transduction (ko04075), pentose and glucuronate interconversions (ko00040), and cyanoamino acid metabolism (ko00460) pathways were significantly enriched with upregulated DEGs and showed lower Q values. The KEGG pathways of plant hormone signal transduction (ko04075), starch and sucrose metabolism (ko00500), plant-pathogen interactions (ko04626), and ribosome biogenesis in eukaryotes (ko03008) were enriched with downregulated DEGs. Among the top 20 KEGG pathways that were enriched with up- and downregulated DEGs, starch and sucrose metabolism (ko00500) and plant hormone signal transduction (ko04075) showed the highest number of DEGs and lowest Q-value, thereby suggesting that these two pathways play important roles during atemoya softening and cracking.

A total of 44,697 DEGs were obtained by pairwise comparison of NT-PC-1/NT-0, NT-PC-2/NT-0, NT-PC-3/NT-0, NT-PC-1/NT-PC-2, and NT-PC-2/ NT-PC-3, and DEG expression profiles were analyzed by STEM. To gain further insights into the biological processes involved in fruit cracking, the DEGs were divided into 25 clusters, of which 11 represented distinct expression patterns. Figure 5 illustrates 11 clusters comprising 26,177 DEGs with significant differential expression $(P$-value $<0.05)$, including seven downregulated patterns (Profiles 0, 1, 2, 3, 4, 9, and 10) and four upregulated patterns (Profiles 15, 16, 24, and 25). The number of DEGs in each profile is shown in Additional file 6. A global description of the biological pathway enriched in each cluster of similarly regulated transcripts using KEGG pathway enrichment is presented in Fig. 5. The genes in clusters 0 and 1 , including a broad range of genes responsible for "Ribosome", "RNA transport", 


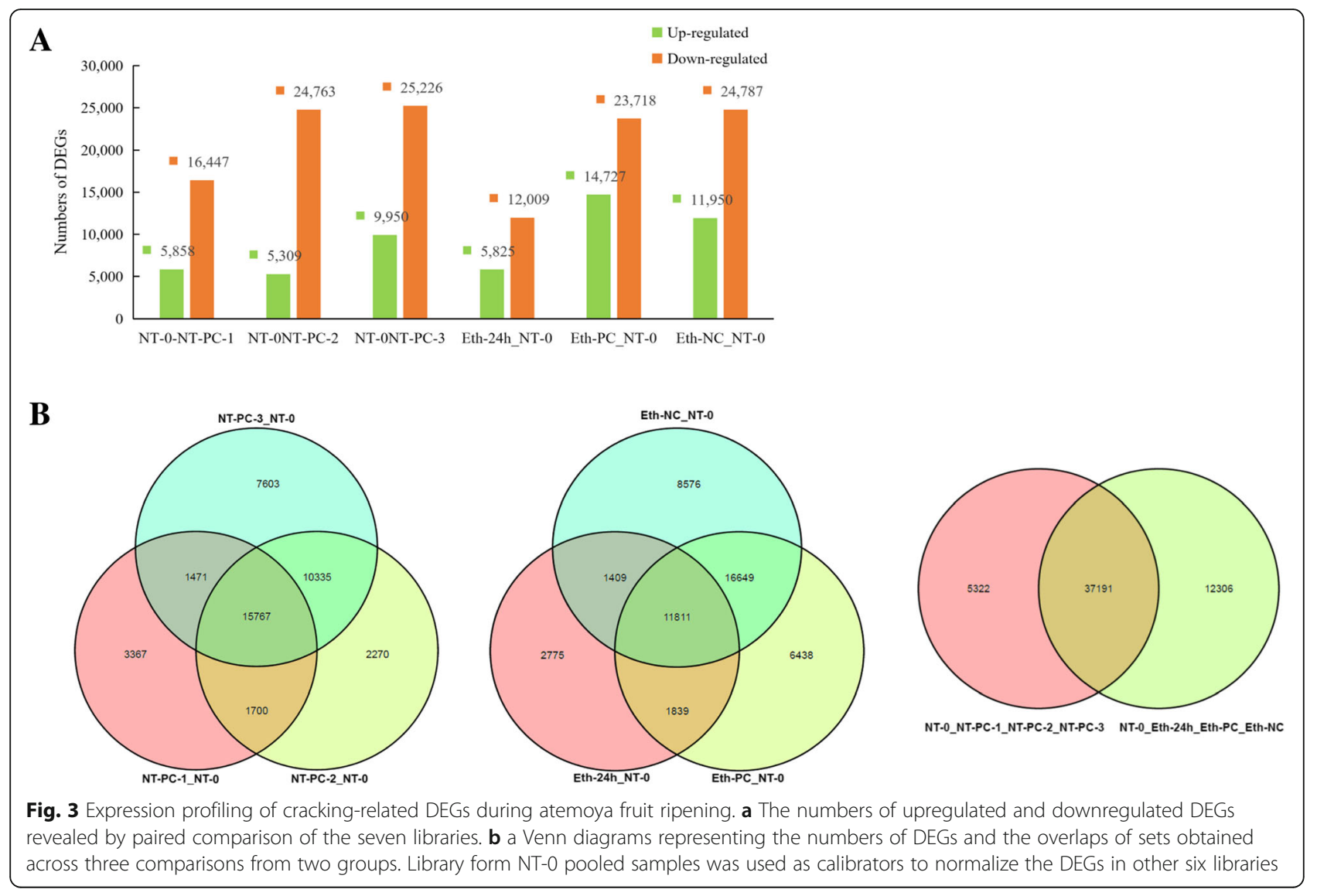

"Spliceosome", "Biosynthesis of amino acids", and "Plant hormone signal transduction" were significantly downregulated from NT-0 to NT-PC-3. The genes from these two profiles mainly involved the degradation of materials in the basal metabolism pathway, which reflected the state of fruit maturation. Clusters 3, 4, and 5 contained genes that were initially downregulated and then subsequently upregulated. These findings suggest that these genes play roles in the later stages of storage (NT-PC-2 and NT-PC-3). These genes mostly belonged to the categories of Amino sugar and nucleotide sugar "metabolism", "Ribosome", "Protein processing in endoplasmic reticulum", "Starch and sucrose metabolism", "Spliceosom", and "Plant hormone signal transduction". The genes induced during the early stages of storage (NT-0 and NT-PC-1) and then subsequently downregulated were grouped into clusters 9 and 10. These genes were mainly involved in the "Carbon metabolism", "Ubiquitin-mediated proteolysis", "Ribosome" and "Peroxisome" pathways and might be responsible for the degradation of materials during earlier stages. Clusters 15, 16, 24, and 25 contained genes that were upregulated during the entire storage stage. These genes are involved in "Starch and sucrose metabolism", "Carbon metabolism", "Plant hormone signal transduction", "Pentose and glucuronate interconversions", and "Ubiquitin-mediated proteolysis". The genes involved in "Starch and sucrose metabolism", "Carbon metabolism", and "Pentose and glucuronate interconversions" are all involved in sugar transformation, thereby confirming that sugar transformation plays an important role in atemoya fruit ripening and cracking, including the conversion of starch into soluble sugars and the degradation of soluble sugars into other forms to generate energy.

\section{Quantitative real-time PCR validation of DEGs}

To further validate our RNA-seq results, we selected 16 unigenes for quantitative real-time PCR (RT-qPCR) analysis. In the KEGG enrichment pathway, the starch and sucrose metabolism pathway and plant hormone signal transduction pathway both ranked higher. Atemoya is the fruit of the starch storage type [25], and the degradation of starch and other sugars is an important metabolic process during ripening, which is closely related to fruit turgor pressure change [8]. In the present research, some cracking-related genes belong to cell wall polysaccharides, which are included in the starch and sucrose metabolism pathway. In addition, plant hormone signal transduction also has been associated with fruit cracking 

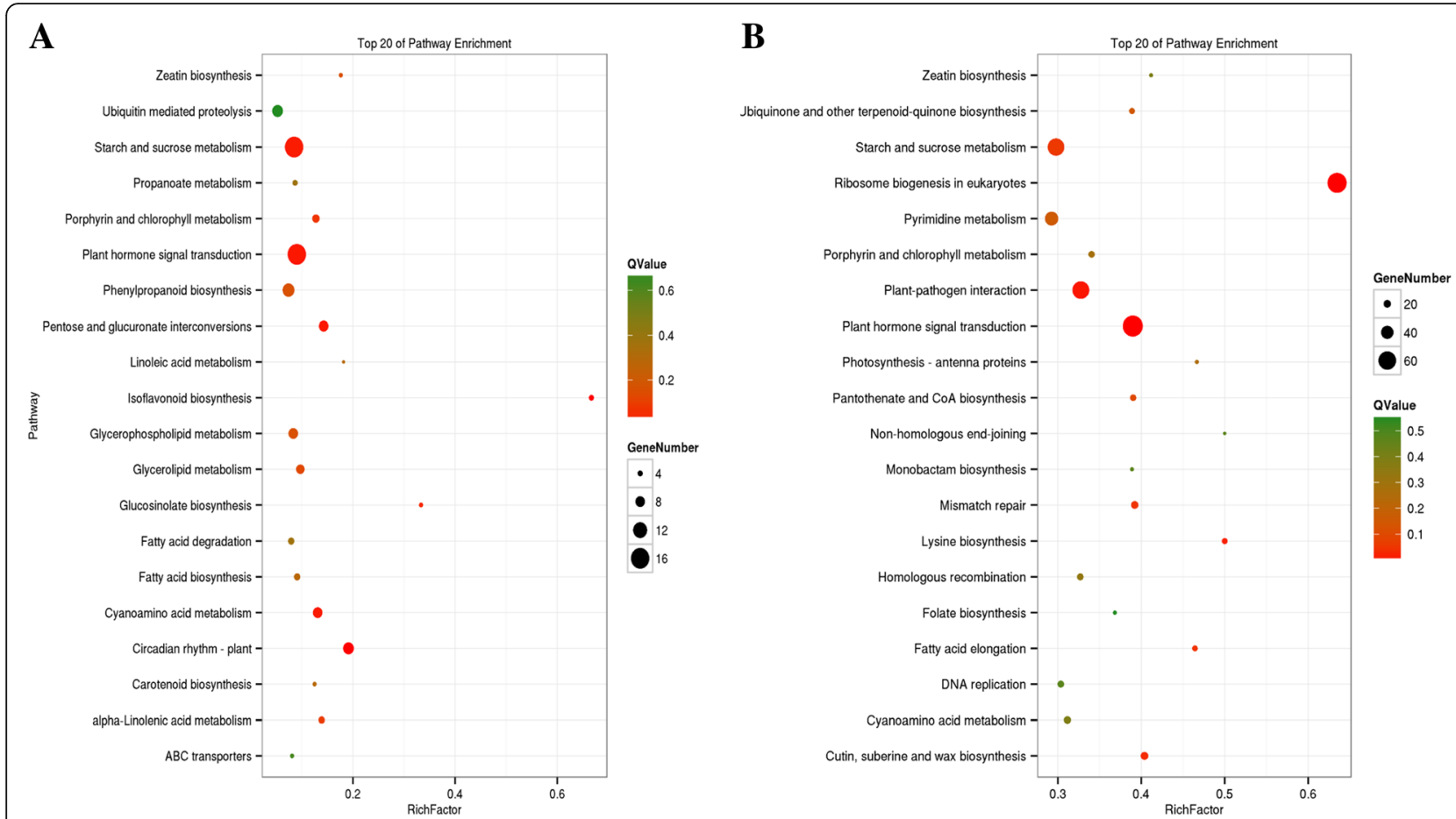

Fig. 4 The top 20 KEGG pathways that are enriched with DEGs. a The KEGG pathways of up-regulated DEGs. b The KEGG pathways of downregulated DEGs. The greater dot indicates the more number of genes, and the deeper red color indicates the smaller Q-value, on the contrary the deeper green color indicates the bigger Q-value

in some fruits, for example Litchi [26]. Thus, we selected 16 genes from these two metabolism pathways.

RNA samples from atemoya pericarp after ethylene treatment and the controls in normal temperature were used as templates. These unigenes are involved in starch and sucrose metabolism, including starch, cellulose, sucrose, and trehalose and plant hormone signal transduction pathways, including ethylene, cytokinin, gibberellin, brassiosteroids, abscisic acid, and jasmonic acid. In the seven sampling stages, the expression profiles of the candidate unigenes coincided with the results of sequencing (Fig. 6a and b) and RNA-seq analysis (Pearson correlation coefficients $\mathrm{R} 2=0.8021$; Fig. $6 \mathrm{c}$ ), thereby validating our transcriptome analysis.

\section{Starch and sucrose metabolism pathway-related DEGs are involved in fruit ripening and cracking}

KEGG pathway enrichment analysis of DEGs of each expression profile determined that the starch and sucrose metabolism pathway was significantly enriched in most clusters (Fig. 5). Table 2 shows the number of the DEGs from 11 differential expression clusters that were involved in the starch and sucrose metabolism pathway from NT-0 to NT-PC-3. A total of 39 DEGs were annotated in the starch and sucrose metabolism pathways, including starch, cellulose, pectin, sucrose, trehalose, maltose, glucose, fructose, and others. Approximately
114 unigenes were selected and used in heat map analysis (Fig. 7). The starch and sucrose metabolism pathway components are shown in Additional file 8.

In the starch metabolism pathway, there were four types of starch synthesis-related DEGs respectively encoding $\operatorname{glg} A$ (starch synthase), $g \lg B$ (1,4-alpha-glucan branching enzyme), $g l g C$ (glucose-1-phosphate adenylyltransferase), and GBSS (granule-bound starch synthase). One $\operatorname{glg} A, 3$ $g l g B, 1 g l g C$, and 2 GBSS were all downregulated from NT-0 to NT-PC-3 and after ethylene treatment during the entire storage period, whereas GBSS (comp126484_c0) was inhibited by ethylene compared to the control (Fig. 7a). Among the starch degradation-related unigenes, three types of unigenes respectively encoding AMYG (glucoamylase), PUL (pullulanase), and glgP (PYG starch phosphorylase) were enriched with a single gene (Table 2). Expression levels of $A M Y G$ and $g l g P$ slightly increased in NT-PC-1 and Eth-24h, and then downregulated during the entire storage period, whereas PUL was upregulated both in normal storage conditions and after ethylene treatment (Fig. 7a). Two main types of starch-degrading enzymes encoding alpha-amylase (AMY) and beta-amylase (BAM) were enriched with six and seven unigenes, respectively (Fig. $7 \mathrm{a}$ and Table 2). There were four upregulated and two downregulated $A M Y$ unigenes, which exhibited similar expression patterns with normal storage conditions and after ethylene treatment. AMY (comp127079_c0) showed the 


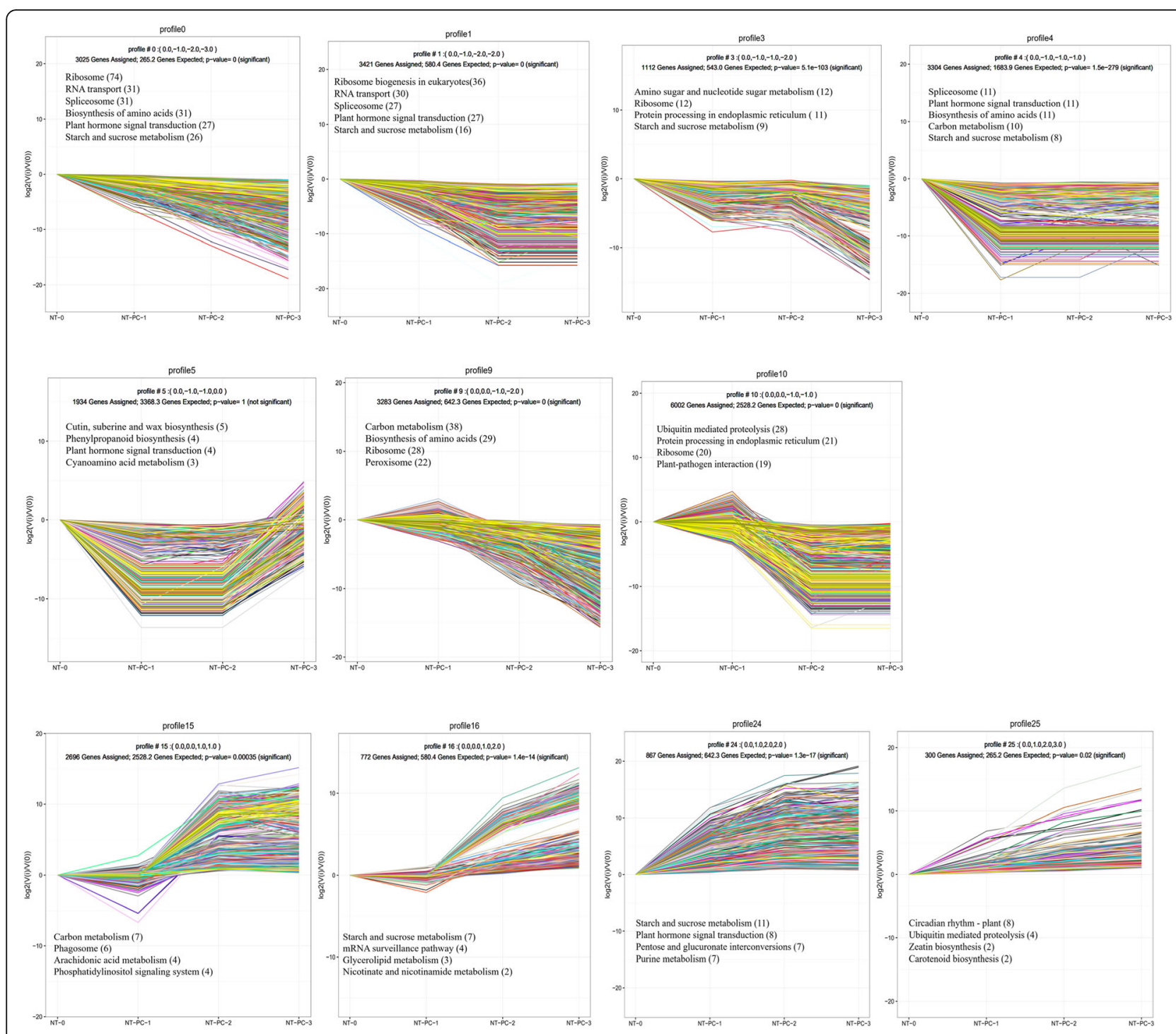

Fig. 5 Cluster analysis of differentially expressed transcripts showing significant changes in expression profiles and KEGG pathway enrichment analysis. Enriched KEGG pathways are listed in each cluster. Numbers in brackets indicates the quantity of DEGs enriched

highest expression levels, and $A M Y$ (comp104952_c0) was the second of six $A M Y$ unigenes that remains upregulated during the entire storage period, suggesting that these might play important roles in starch degradation. Seven $B A M$ unigenes were enriched, of which five were upregulated, two were downregulated both with normal storage conditions and after ethylene treatment (Fig. $7 \mathrm{a}$ and Table 2). BAM (comp127079_c0) and BAM (comp127079_c1) had very high expression levels, higher than $A M Y$ (comp127079_c0) (Fig. 7a), whereas the two unigenes belonged to different fragments of the same gene, as indicated by BLAST. Compared to $A M Y, B A M$ might be the main enzyme of starch degradation. Four DEGs encoded $g l g X$ (glycogen-debranching enzyme), which included two downregulated and two upregulated genes both in normal storage conditions and after ethylene treatment. $G \lg X$ (comp129231_c0) induced higher expression with ethylene compared to the control. In the starch metabolism pathway, all of the synthetase genes were downregulated during fruit cracking, whereas most of the degradation-related genes, including $A M Y, B A M, P U L$, and $g l g X$, were upregulated. Together, these findings suggest that starch is extensively degraded during fruit ripening and cracking.

The pericarp is directly involved in fruit cracking, and the components of the pericarp are closely related to the development of cracking resistance. Most of the cell wall components are polysaccharides, such as cellulose and pectin. Enriched cellulose-related genes mainly encode cellulose-degrading enzymes, including two kinds of genes, namely, endoglucanase and beta-glucosidase. Two endoglucanase genes and 16 beta-glucosidase genes were enriched, of which 1 endoglucanase and 2 beta-glucosidase 
A
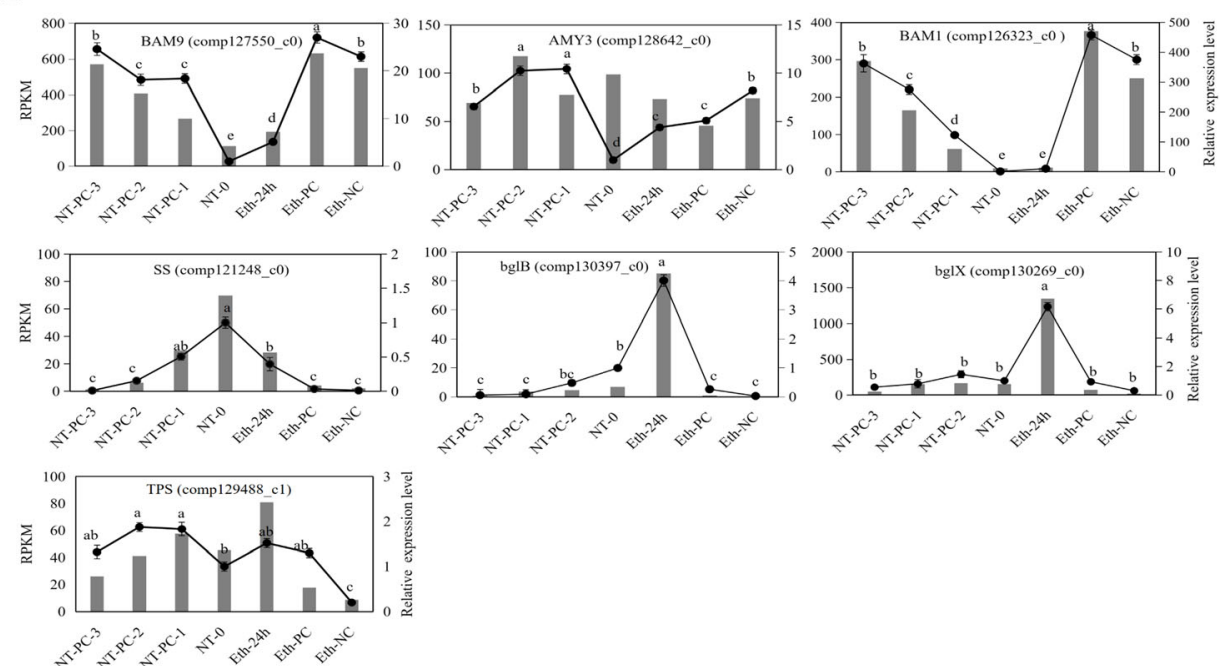

B

Plant hormone signal transduction related-genes
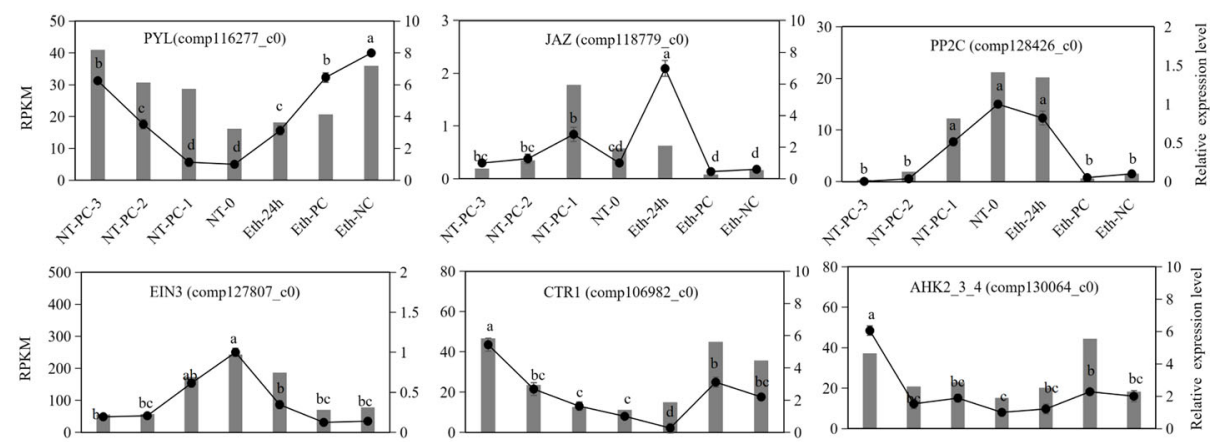

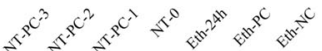

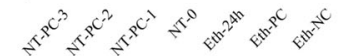

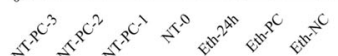
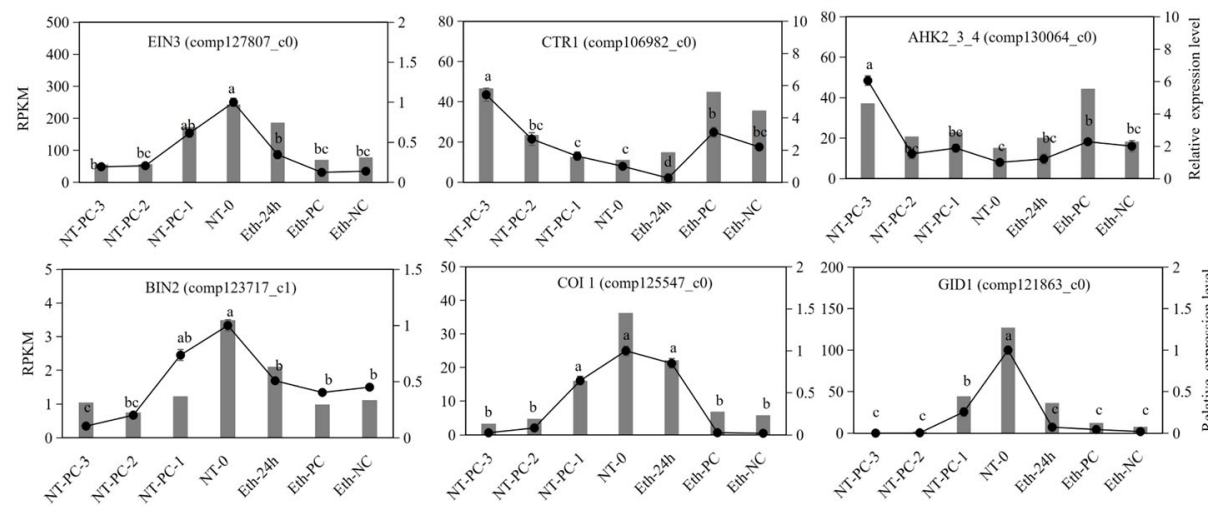

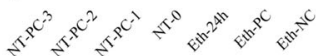

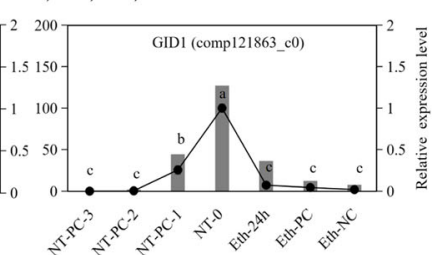

C

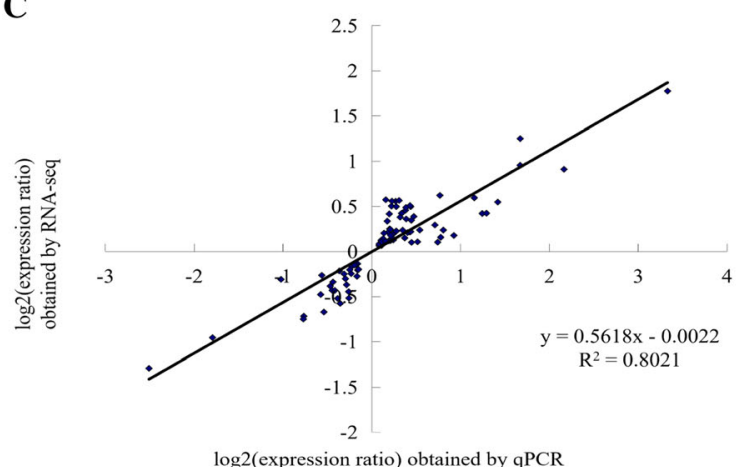

Fig. 6 (See legend on next page.) 
(See figure on previous page.)

Fig. 6 Candidate unigene expression levels verification and coefficient analysis of fold-change data between RT-qPCR and RNA-seq. a Transcript levels and RT-qPCR results of 7 selected genes from RNA-sequencing, which belonged to the starch and sucrose metabolism pathway. $\mathbf{b}$ Transcript levels and RT-qPCR results of nine selected genes from RNA-sequencing, which belonged to plant hormone metabolism pathway. The 16 unigenes were $B A M 9, A M Y 3, B A M 1$, SS, blgB, blgX, COI1, TPS, GID1, PYL, JAZ, PP2C, EIN3, CTR1, AHK2-3-4, and BIN2, and their full names are shown in the "Abbreviations" sections. The left $y$-axis shows the relative gene expression levels analyzed by qPCR (black lines). The right $y$-axis indicates the corresponding expression data of RNA-seq (gray histogram). The x-axis represents sampling time. The $C T$ value of each gene was the average of three technical replicates with the standard error indicated. Significant difference of relative expression value in the different sampling times estimated by Duncan's test was reported on the graphics ( $p$-value $<0.05$ ). Means labeled by the same letter are not significantly different. c Scatterplots were generated by the log 2 expression ratios from RNA-seq (y-axis) and RT-qPCR (x-axis)

genes were upregulated and 1 endoglucanase and 14 beta-glucosidase were downregulated both with normal storage conditions and after ethylene treatment (Fig. 7c and Table 2). In 14 downregulated unigenes, the expression of 5 beta-glucosidase genes, including comp119470_c2, comp121604_c0,comp129279_c0,comp130397_c0, and comp130269_c0, were induced to different degrees using Eth-24 h, whereas these were continuously downregulated from NT-0 to NT-PC-3 with normal storage conditions (Fig. 7c).

An enrichment of pectin metabolism-related unigenes was also observed, except for GAUT (alpha-1,4- galacturonosyltransferase) that participates in pectin synthesis, as well as $P E$ (pectinesterase), $P G$ (polygalacturonase), and $P E L$ (pectate lyase), which are involved in pectin degradation. Ten unigenes encoding GAUT were differentially expressed, of which three presented upregulated profiles, and the rest were downregulated both under normal storage conditions and after ethylene treatment. There were $11 P E, 12 P G$, and 6 PEL unigenes that were respectively enriched, of which 8,12 , and 5 were upregulated both in normal storage conditions and after ethylene treatment (Fig. 7b and Table 2). Two PE unigenes (comp124968_c0 and comp127036_c0), three PG unigenes (comp120584_ c0,comp123401_c0, and comp120584_c1), and two PEL unigenes (comp122322_c0 and comp116480_c1) presented higher expression than other pectin-degrading enzymes genes both under normal storage conditions and after ethylene treatment, but there were no obvious differences between the controls and ethylene treatment (Fig. 7b). An enrichment of pectin-degrading enzymes was also observed, and most of these were upregulated, which suggested that pectin degradation was intimately linked to fruit ripening and cracking. Several unigenes encoding TPS (trehalose 6-phosphate synthase/phosphatase), malQ (4-alpha-glucanotransferase), scrK (fructokinase), SS (sucrose synthase), 1,3-beta-glucan synthase, UXS1 (UDP-glucuronate decarboxylase), and other sugar metabolism-related genes are involved in trehalose, maltose, sucrose, fructose, and glucose metabolism pathways, which ware responsible for converting all kinds of polysaccharides into monosaccharides. The contribution of different kinds of sugars to osmotic pressure varies, with monosaccharides providing the largest contribution, and the rate of conversion of polysaccharides to monosaccharides can influence osmotic pressure. Therefore, enrichment analysis of sugar-related genes may identify the major sugars that are metabolized in atemoya fruits and their possible effects on changes in osmotic pressure during fruit cracking. According to enriched sugar related-genes, trehalose, sucrose, fructose, glucose, and maltose are the major soluble sugars in sugar metabolism during fruit ripening. Most of these were downregulated during the entire storage period; nevertheless, some of these had high expression levels at NT-0, NT-PC-1 and Eth-24h, e.g., SS (comp122833_c0), scrK (comp122886_ c1), TREH (alpha,alpha-trehalase)(comp124814_c1), UDPglucuronate 4-epimerase (comp127368_c1), and UXS1 (comp121018_c0) (Fig. 7d and e). The above results only preliminarily identified the major types of sugar, and the next step is to quantify the contents of the major sugars at each stage to determine the conversion rate of sugars and their effect on osmotic pressure.

\section{Changes in starch and pectin contents and their metabolism-related genes expression profiles}

Figure $8 \mathrm{a}$ shows that starch content decreased during fruit storage, and ethylene treatment accelerated starch degradation. Protopectin content in the pericarp without treatment increased until day 4 , whereas it decreased after day 1 with ethylene treatment (Fig. 8b), which indicated that ethylene inhibited the synthesis of the protopectin. Figure $8 \mathrm{c}$ shows that soluble pectin content increased during fruit storage, and ethylene treatment enhanced soluble pectin content compared to no treatment, which suggests that ethylene treatment accelerates pectin degradation.

To shed light on which genes play important roles in starch and cell wall polysaccharide metabolism pathways during fruit cracking, the expression profiles of 12 unigenes were analyzed. Among these, $A M Y 3$ (comp12864 2_c0), BAM1 (comp126323_c0), and BAM9 (comp127550 _c0) that were validated by RT-qPCR (Fig. 6a). Nine starch-degrading enzymes, namely, $A M Y 1$ (comp104952 c0), AMYG (comp124474_c0), BAM3 (comp127079_c0), PUL (comp128177_c0), glgX (comp129444_c0), glgP (comp 124413_c0), AMY3, BAM1, BAM9, and one starch synthesis gene $\operatorname{glg} B$ (comp125073_c0), were selected (Fig. 9a). 
Table 2 Numbers of DEGs involved in starch and sugar metabolism pathways during cracking of atemoya pericarp

\begin{tabular}{|c|c|c|}
\hline & NT-0_NT-PC-1_NT-PC-2_N & VT-PC-3 \\
\hline & Down regulation & Up regulation \\
\hline & Profile $0,1,2,3,4,9,10$ & Profile $15,16,24,25$ \\
\hline Starch & & \\
\hline glgA; starch synthase [EC:2.4.1.21] & 1 & 0 \\
\hline GBSS; granule-bound starch synthase [EC:2.4.1.242] & 2 & 0 \\
\hline glgB; 1,4-alpha-glucan branching enzyme [EC:2.4.1.18] & 3 & 0 \\
\hline glgC; glucose-1-phosphate adenylyltransferase [EC:2.7.7.27] & 1 & 0 \\
\hline glgP; starch phosphorylase [EC:2.4.1.1] & 1 & 0 \\
\hline AMYG; glucoamylase [EC:3.2.1.3] & 1 & 0 \\
\hline AMY; alpha-amylase[EC 3.2.1.1] & 2 & 4 \\
\hline BAM; beta-amylase [EC:3.2.1.2] & 2 & 5 \\
\hline glgx; glycogen debranching enzyme [EC:3.2.1.196] & 2 & 2 \\
\hline PUL; pullulanase [EC:3.2.1.41] & 0 & 1 \\
\hline Cellulose & & \\
\hline endoglucanase [EC:3.2.1.4] & 2 & 0 \\
\hline beta-glucosidase [EC:3.2.1.21] & 8 & 2 \\
\hline bglB; beta-glucosidase [EC:3.2.1.21] & 3 & 0 \\
\hline bgIX; beta-glucosidase [EC:3.2.1.21] & 2 & 1 \\
\hline Pectin & & \\
\hline GAUT; alpha-1,4-galacturonosyltransferase [EC:2.4.1.43] & 7 & 3 \\
\hline$P E ;$ pectinesterase [EC:3.1.1.11] & 3 & 8 \\
\hline PG; polygalacturonase [EC:3.2.1.15] & 0 & 12 \\
\hline PEL; pectate lyase [EC:4.2.2.2] & 1 & 5 \\
\hline Sucrose & & \\
\hline sucrose synthase [EC:2.4.1.13] & 2 & 0 \\
\hline yihQ; alpha-glucosidase [EC:3.2.1.20] & 0 & 1 \\
\hline Trehalose & & \\
\hline TPS; trehalose 6-phosphate synthase/phosphatase [EC:2.4.1.15 3.1.3.12] & 8 & 0 \\
\hline otsA; trehalose 6-phosphate synthase [EC:2.4.1.15] & 0 & 0 \\
\hline otsB; trehalose 6-phosphate phosphatase [EC:3.1.3.12] & 1 & 2 \\
\hline TREH, treA, treF; alpha,alpha-trehalase [EC:3.2.1.28] & 0 & 1 \\
\hline Maltose & & \\
\hline malQ; 4-alpha-glucanotransferase [EC:2.4.1.25] & 0 & 3 \\
\hline Fructose & & \\
\hline scrk; fructokinase [EC:2.7.1.4] & 9 & 0 \\
\hline HK; hexokinase [EC:2.7.1.1] & 1 & 0 \\
\hline Glucose & & \\
\hline 1,3-beta-glucan synthase [EC:2.4.1.34] & 0 & 1 \\
\hline glucan 1,3-beta-glucosidase [EC:3.2.1.58] & 0 & 1 \\
\hline Others & & \\
\hline UXS1, uxs; UDP-glucuronate decarboxylase [EC:4.1.1.35] & 5 & 1 \\
\hline XYL4; beta-D-xylosidase 4 [EC:3.2.1.37] & 2 & 0 \\
\hline UDP-glucuronate 4-epimerase [EC:5.1.3.6] & 5 & 0 \\
\hline UGDH, ugd; UDPglucose 6-dehydrogenase [EC:1.1.1.22] & 1 & 0 \\
\hline
\end{tabular}


Table 2 Numbers of DEGs involved in starch and sugar metabolism pathways during cracking of atemoya pericarp (Continued)

\begin{tabular}{llc}
\hline & NT-0_NT-PC-1_NT-PC-2_NT-PC-3 \\
\cline { 2 - 3 } & Down regulation & Up regulation \\
\hline ENPP1_3; ectonucleotide pyrophosphatase/phosphodiesterase family member 1/3 [EC:3.1.4.1 3.6.1.9] & 1 & 0 \\
pmm-pgm; phosphomannomutase / phosphoglucomutase [EC:5.4.2.8 5.4.2.2] & 0 & 2 \\
pgmB; beta-phosphoglucomutase [EC:5.4.2.6] & 2 & 1 \\
\hline
\end{tabular}

Among these, except for $g l g P$ and $A M Y G$, the expression of the other degrading enzyme genes were significantly upregulated, whereas that of the synthesis genes decreased during fruit cracking after ethylene treatment and in the controls. $A M Y 1, B A M 3, B A M 9$, PUL, and $g l g X$ were all upregulated and exhibited higher expression levels with ethylene treatment compared to without treatment, which could be responsible for accelerating starch degradation after ethylene treatment. With ethylene treatment and in the controls, $A M Y 1$ and $B A M 3$ were significantly expressed, which indicated these two genes are the key genes of starch degradation.

GAUT is the key pectin synthesis enzyme [27]. High amounts of GAUT (comp126456_c0) accumulated during the first three stages, with a reduction at the fourth stage with controls and ethylene treatments, and higher expression levels under controls compared to ethylene treatment, whereas its expression profile coincided with changes in protopectin content, which could account for protopectin synthesis during fruit cracking and softening (Fig. 9b). PE (comp124968_c0), PG (comp130329_c0), and PEL (comp122322_c0) were upregulated with or without ethylene treatment, which indicated that these three genes play important roles in pectin degradation. Among these, $P E$ was significantly upregulated by ethylene, and thus might be the key gene involved in pectin degradation (Fig. 9b). Beta-glucosidase (comp127164_c0) was upregulated by ethylene, indicating that it could be a major cellulose degradation enzyme (Fig. 9b).

\section{Discussion}

Several factors influence fruit cracking, including genetics, morphology, physiology, environmental conditions (e.g., temperature, wind and light), and orchard management (e.g., irrigation and nutrition). Furthermore, fruit cracking is a quantitative trait that is controlled by several genes [8]. To generate molecular evidence and elucidate the mechanism underlying fruit cracking, the present study attempted to identify fruit cracking genes in atemoya during postharvest storage. To circumvent the unavailability of an atemoya reference genome, we utilized the pericarp of cracking atemoya fruits for RNA-seq in the de novo assembly of a reference transcriptome. Our assembly contains a total of 64,069 unigenes with a mean size of $670 \mathrm{bp}$. Approximately 25,074 unigenes were annotated to public protein databases. We performed a pairwise comparison and identified 42,513 DEGs between NT-0 and NT-PC-1, NT-PC-2, and NT-PC-3 and 49,497 DEGs between NT-0 and Eth-24 h, Eth-PC, and Eth-NC. KEGG pathway enrichment analysis of all DEGs and DEGs of each distinct expression patterns indicated that the starch and sucrose metabolism pathway was significantly enriched.

The present study determined that in cherimoya, carbohydrates are mainly stored in the form of starch [28]. Scanning electron microscope (SEM) analysis of AP atemoya indicated numerous starch granules in the fruit tissues [29]. Thus, the cells of the atemoya fruit not only store starch but also use this to maintain cell turgor [30]. Mo et al. [11] suggested that the firmness of Annona fruits dramatically decreases with ripening, possibly due to transformation of starch into soluble sugars. Wills et al. [31] showed that the initiation of cracking coincides with the decline in about one-fifth of the starch level in fruits. Paull [14] suggested that atemoya cracking commonly occurs during postharvest because of changes in osmotic and subsequent turgor, which are related to the production of neutral sugars during ripening, which leads to the movement of water from the skin and receptacle to the flesh. Previous studies have revealed that starch degradation induces changes in fruit turgor, and fruit softening and cracking are closely related; however, these were mainly observed at the physiological level. In the present study, transcriptome sequencing revealed that fruit cracking is strongly associated with a significant enrichment of several starch metabolism genes, including 7 starch synthase genes and 17 starch degrading enzymes. At the transcriptional level, the transformation of starch to soluble sugars may be one of the most important processes in fruit softening and cracking. Unigenes encoding starch synthase were all downregulated, whereas starch degradation genes were mostly upregulated. For example, $A M Y 1$ (comp104952_c0) and BAM3 (comp127079_c0) were significantly induced and highly expressed during fruit cracking, which suggests that these are key to starch degradation, and simultaneously showed that starch is rapidly decomposed by amylase during postharvest. We also showed that starch degradation is related to fruit cracking, which coincides with the results of previous studies on physiological metabolism. 


\section{A}

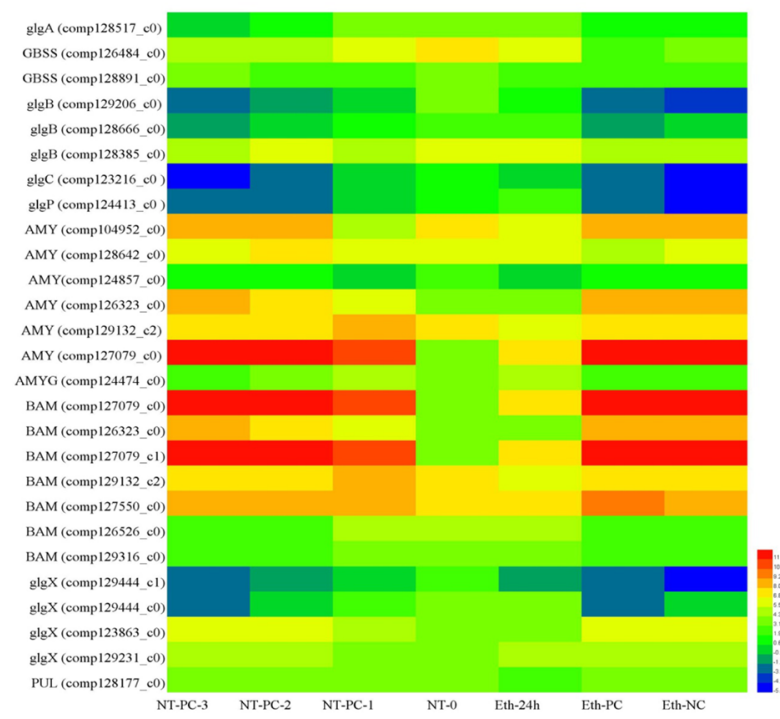

C

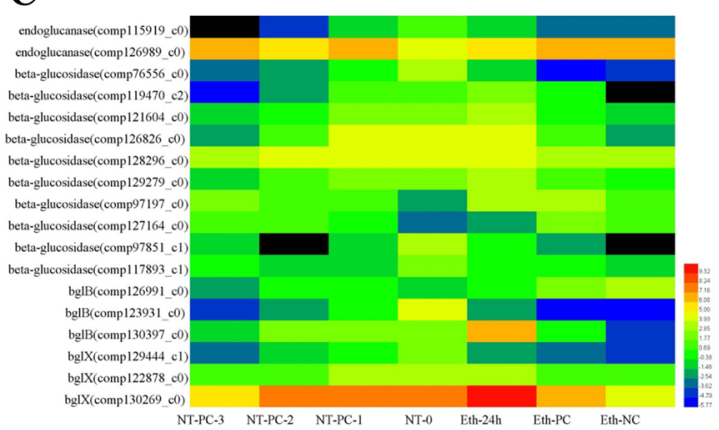

B

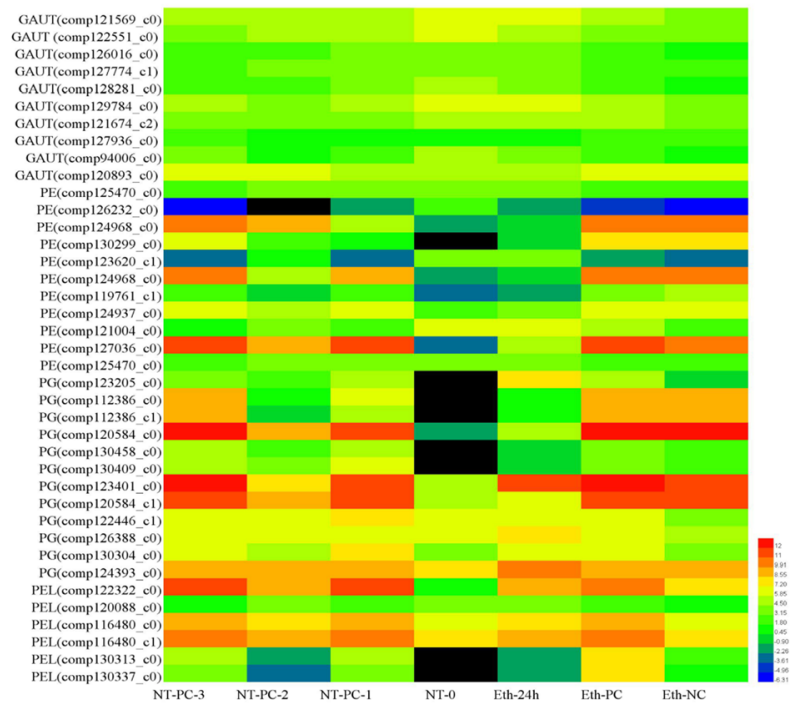

D

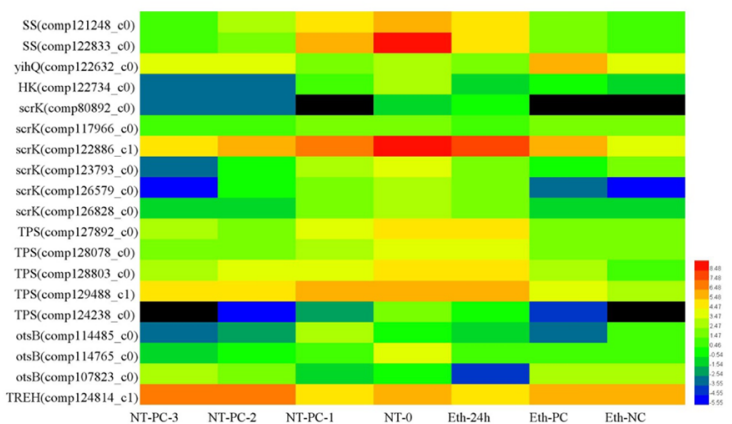

$\mathbf{E}$

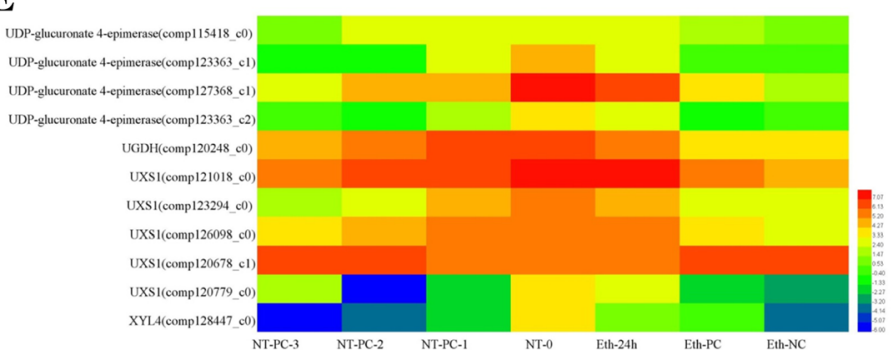

Fig. 7 Heat map diagram of the expression patterns for DEGs annotated in the starch and sucrose metabolism pathway analyzed by KEGG. a Starch metabolism-related genes. b Pectin metabolism-related genes. c Cellulose metabolism-related genes. d Trehalose, sucrose, fructose, and glucose metabolism-related genes. e Other metabolism-related genes in the starch and sucrose metabolism pathway. Abbreviated names and full names of all of the genes in the heat map are listed in Table 2. The data on gene expression levels were normalized. Each column represents an experimental sample (e.g., NT-0, NT-PC-1, NT-PC-2, NT-PC-3, Eth-24 h, Eth-PC, and Eth-NC), and each row represents a gene. The color scale at the right represents the log-transformed RPKM value, and expression differences are shown in different colors. Red indicates high expression, and green represents low expression

Soluble sugar content affects fruit turgor, thereby leading to fruit cracking. Previous studies on sweet cherry, wax apple, litchi, grapes, and other fruits have confirmed that the soluble sugar content is higher and osmotic potential is lower in cracking fruits than those without cracking [20,32-34], which indicates that water, soluble sugar, and osmotic potential are closely related to fruit cracking. Lu and Lin [20] reported that the TSS content and total titratable acid levels were both $20 \%$ higher in cracked fruits than in non-cracked fruits of wax apple. Furthermore, when its turgor pressure was $60 \%$ higher, an increase in TSS content and total titratable acid levels 

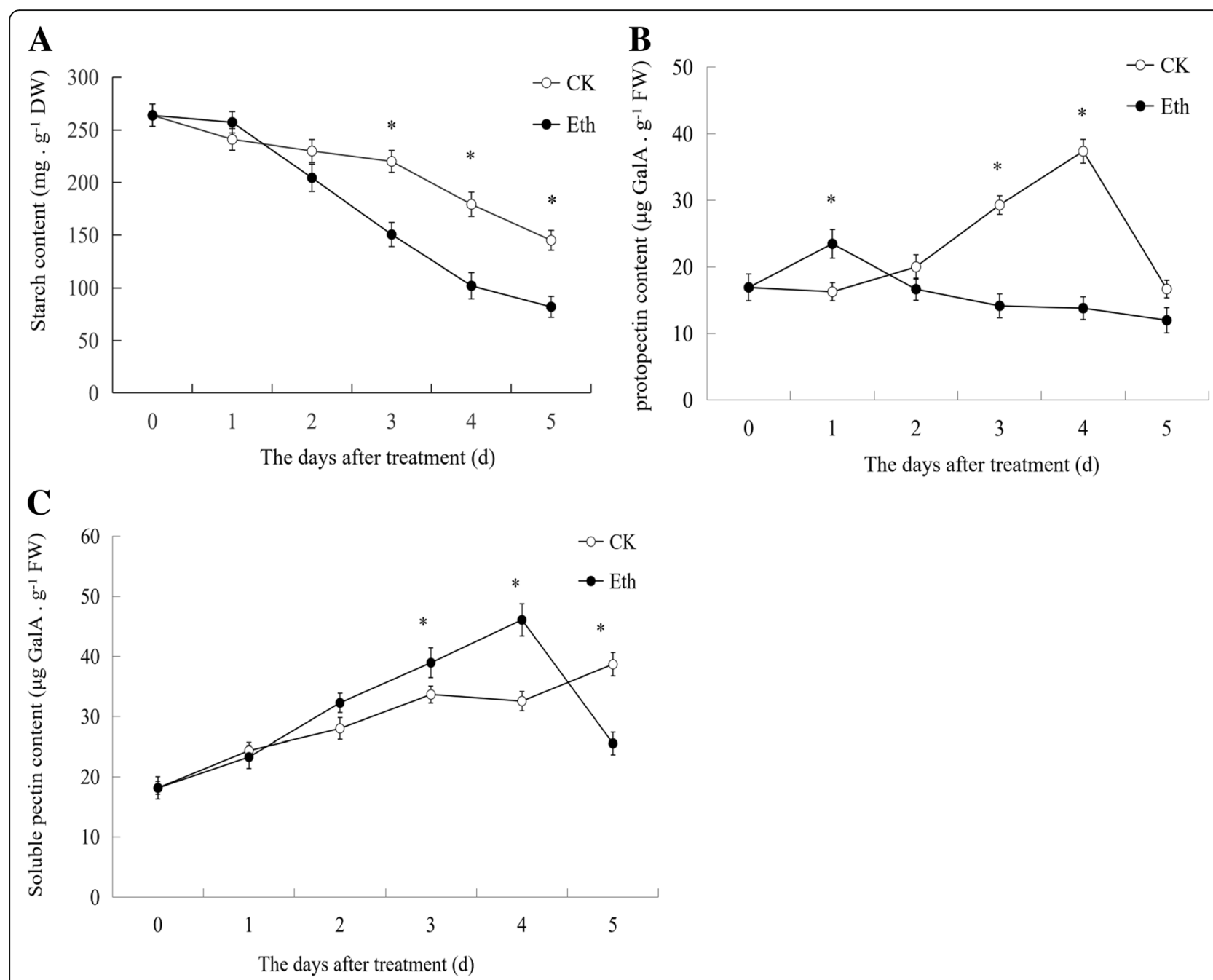

Fig. 8 Analysis of starch, protopectin, and soluble pectin contents with or without ethylene treatment during atemoya storage. a Starch (b) Protopectin (c) Soluble pectin. Vertical bars represent the standard error of three biological replicates. Significant differences in starch, protopectin, and soluble pectin content for each day between no treatment (CK) and ethylene treatment (Eth) estimated using the t-test is shown in the graph (p-value $<0.05)$. Asterisks $\left(^{*}\right)$ indicate significant differences in starch, protopectin, and soluble pectin content between CK and Eth at the same day

during fruit maturation was observed, which in turn leads to a decrease in tissue osmotic potential. Considine and Kriedemann [32] provided direct evidence for the importance of fruit soluble solids, wherein cracking in grapes occurs when these are submerged in solutions of low osmotic potential. Atemoya fruits are of the starch storage type [25], and the rate and ability to undergo starch degradation during postharvest is directly related to soluble sugar content, which in turn directly affects fruit turgor [8]. Therefore, factors influencing starch degradation rate, such as temperature and ethylene, could also influence the degree of fruit cracking. Under low temperature, TSS content of cherimoya fruits slowly increased, whereas no detectable change in $\mathrm{pH}$ value was observed [35, 36], which suggests that low temperature treatment of cherimoya results in a decrease in the rate of starch hydrolysis and monosaccharide concentration, and these findings agree with the results of our investigation in atemoya [37]. Gutiérrez et al. [28] determined using ultrastructural analysis of fruits that starch grains do not break down, and the cell walls remain intact after $6 \mathrm{~d}$ of storage at $4{ }^{\circ} \mathrm{C}$. Except for low temperature, our previous research suggests that ethylene could significantly accelerate cracking and ripening in atemoya, and the pericarp cracks first after $2 \mathrm{~g} / \mathrm{kg}$ of ethephon treatment, which is different from the control treatment [22]. This research found that ethylene accelerated starch degradation and enhanced the content of soluble pectin, compared to the control, suggesting that ethylene accelerates fruit cracking and ripening. Transcriptome analysis suggests a strong association between the degradation of starch, pectin, and cellulose, and the upregulation of AMY1 (comp104952_c0), BAM3 (comp127079_c0), BAM9 (comp127550_c0), PUL (comp128177_c0), glgX 


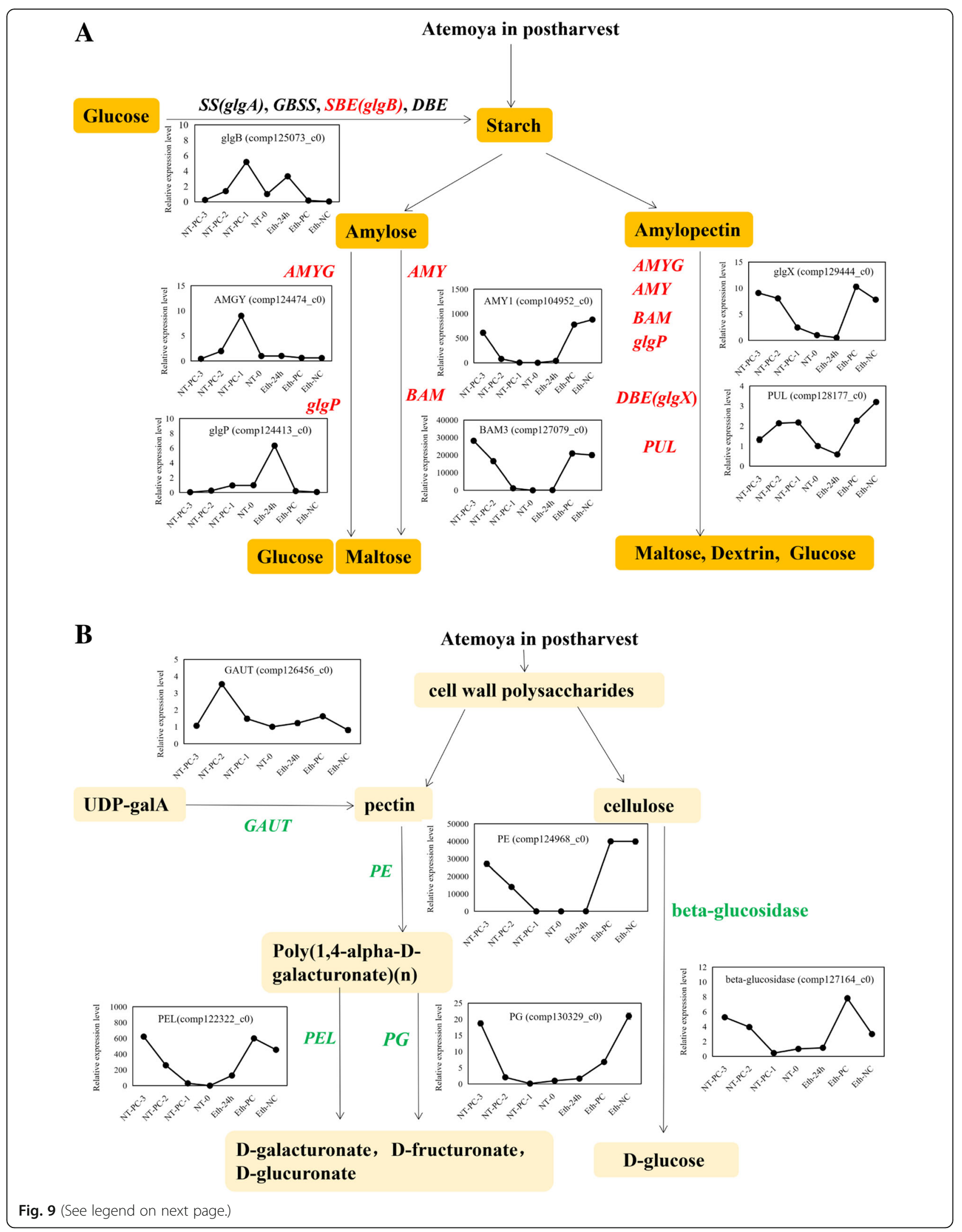


(See figure on previous page.)

Fig. 9 Simplified scheme of the polysaccharide metabolism pathway. (A) Starch metabolism pathway; genes examined are in red italic bold letters, which include glgB, AMY1, BAM3, AMYG, glgP, glgX, and PUL.(B) Pectin and cellulose metabolism pathway. Genes examined are in green italic bold letters, which include GATU, PE, PG, PEL, and beta-glucosidase. The full names of the genes are shown in Table 2 and the part of the "Abbreviations" section

(comp129444_c0), PE (comp124968_c0) and beta-glucosidase (comp127164_c0). In addition, the ethylene treatment not only promoted the increase of soluble pectin, but also inhibited the synthesis of the propectin, which could explain why the pericarp cracks first after ethylene treatment. At the transcriptional level, GAUT (comp126456_c0) is mainly involved in the synthesis of the propectin. The above research showed that the external environment temperature and ethylene treatment could be directly related to the time and extent of fruit cracking by regulating the metabolism of starch and cell wall polysaccharides.

Previous studies have found that starch is transformed into various kinds of soluble sugars such as disaccharides (e.g., sucrose) and monosaccharides (e.g., fructose and glucose). Paull et al. [38] reported that in soursop, sucrose content increased by four-fold at maturity, and the highest concentration was observed at 3 days after harvest, which subsequently decreased to $40 \%$. Fructose and glucose content then slowly increase until 5 days after harvest. The ratio of sucrose, glucose, and fructose at the edible ripe stage was 4.3:3.0:3.2 [39]. However, in cherimoya (Annona cherimola Mill. cv. 'Fino de Jete'), starch is mainly converted into glucose and fructose in equimolar concentrations, with little and transient accumulation of sucrose [28, 40]. In our research, some genes that were involved with sucrose, fructose, and glucose were enriched, such as sucrose-metabolizing enzymes, $S S$ and alpha-glucosidase $(Y i h Q)$, and fructose metabolism-related enzymes, scrK, and hexokinase ( $H K$ ), glucose metabolism genes, and glucan 1,3-beta-glucosidase. Furthermore, trehalose metabolism-related genes, including eight trehalose 6-phosphate synthase (TPS) genes, three trehalose 6-phosphate phosphatase (otsB) genes, one TREH gene, and three maltose metabolismrelated genes malQ, were enriched. The degradation of starch produces maltose, whereas previous studies have shown that trehalose is rarely involved in starch transformation of Annona fruits. Recent studies have observed that trehalose in plants is also involved in metabolism and gene expression regulation and affects plant growth, development, and responses to changes in the external environment [41, 42]. Trehalose is a typical stress metabolism and protective substance, and when the organism is under stress, it can quickly accumulate trehalose $[43,44]$. The role of trehalose during atemoya postharvest requires further investigation.
Researchers have found that some cell wall-related genes, which include EXP, XET, $P G$, and $P E$, also play roles in fruit cracking $[8,21]$. Shen et al. [19] suggested that during cracking and ripening of fruits in the field, the expression of AsEXP1 and AsEXP3 are relatively high, whereas that of AsEXP2 is relatively weak. Wang et al. [17] reported that the differential expression of LcEXP1 and LcEXP2 in the litchi pericarp is related to fruit growth and resistance to crack formation. Our previous research [22] compared cracking-susceptible cultivars with cracking- resistant cultivars of atemoya during postharvest storage and we found that EXP1 is more relevant to fruit maturity and softening. EXP2, EXP3, and $P E$ were more closely related to fruit cracking, whereas $X E T 1, X E T 2$, and $X E T 3$ might be related to fruit softening and are unlikely key genes of fruit cracking [22]. Lu and Lin [20] reported a $131 \%$ increase in PG activity in cracked fruits compared to non-cracked wax apple fruits. In the present research, transcriptome sequencing analysis found that except for $12 P G$ and $11 P E$, another pectin degradation-related gene, $6 P E L$, was enriched, suggesting that pectin decomposition is also an important polysaccharide metabolism process in atemoya fruit cracking. At the physiological level, combining part of the gene expression evidences from previous researches with our RNA-seq results strengthens our theory that atemoya fruit cracking is caused by the transformation of starch into soluble sugars that in turn increases turgor pressure, thereby resulting in the rupture of cells and tissues, as well as the degradation of cell wall polysaccharides such as pectin and cellulose, ultimately decreasing cell wall toughness, which is exhibited as cracking of the pericarp and flesh.

RT-qPCR analysis indicated an enrichment of numerous starch, pectin, and cellulose metabolism-related genes, which were predominated by AMY1 (comp10495 2_c0), BAM3 (comp127079_c0), PUL (comp128177_c0), glgX (comp129444_c0), PE (comp124968_c0), PG (comp1 30329_c0), PEL (comp122322_c0), and beta-glucosidase (comp127164_c0). Since the present study was designed as a preliminary validation, additional studies looking into the kind of relationship between these genes and fruit cracking are warranted. In addition, fruit cracking is a complex physiological phenomenon in that no single gene directly controls the process. However, transcriptome sequencing has provided molecular evidence that starch and cell wall polysaccharides may both be involved in fruit cracking, as well as useful genetic resources for screening additional fruit cracking-related genes. 
In addition to the important role of starch and sucrose metabolism pathway in atemoya fruit cracking, studies have shown that plant hormone signal transduction pathways are also involved in fruit cracking. Spraying different concentrations of gibberellin on cherries, pomegranates, and litchi can reduce fruit cracking rate [45-47]. In litchi and pomegranate, ABA content in pericarp is higher in cracking fruits than in the healthy fruits $[48,49]$, and spraying ABA increases the fruit cracking rate of litchi [11]. These researchers suggest that spraying GA can reduce fruit cracking, but spraying ABA can increase fruit cracking in some fruits. Li et al. [26] performed comparative transcriptomic analyses of non-cracking and cracking litchi fruits and found that plant hormone signal transduction pathway-related genes have been enriched, including GA and ABA. They believe that lower GA levels and higher ABA levels in pericarp lead to slower pressure expansion of the pericarp, so that aril cells produce more turgor against the skin, thereby leading to fruit cracking. In our results, the enriched number of DEGs of the plant hormone signal transduction pathway ranked fourth in all metabolism pathways, consisting of a total of 154 DEGs, including gibberellin, abscisic acid, and cytokinins (Additional 4), whereas we did not focus on plant hormone-related genes in this study, and thus their role in fruit cracking requires further investigation.

\section{Conclusions}

In summary, seven sets of transcriptome data comprising 64,069 unigenes in the atemoya pericarp were generated by Illumina sequencing. Both KEGG pathway enrichment analysis of DEGs and material content analysis confirmed that starch decomposition into soluble sugars and cell wall polysaccharides metabolism are closely related to the ripening and cracking of the AP atemoya. RT-PCR analysis indicated that the differential expression of genes during different cracking stages of the atemoya fruit influences starch and pectin content. In addition, plant hormone signal transduction pathway-related genes were significantly enriched, suggesting their role in fruit ripening and cracking. The present study may serve as a valuable resource for future atemoya genomic studies and may also benefit investigations involving other closely related agricultural species. The DEG dataset may also provide useful candidate genes for the elucidation of the mechanism underlying fruit cracking in atemoya and other fruits.

\section{Methods}

Plant materials and experimental design

In the present study, AP atemoya fruits were collected from the Base of National Field Genebank for Tropical
Fruit at South Subtropical Crops Research Institute, Chinese Academy of Tropical Agricultural Sciences, Zhanjiang, Guangdong, China. The orchard is located at $21^{\circ} 12^{\prime}$ $\mathrm{N}, 110^{\circ} 4^{\prime} \mathrm{E}$. Material collection was conducted in accordance with local legislation, and there was no need for permission from other organizations. We complied with the Convention on the Trade in Endangered Species of Wild Fauna and Flora. Fruits were harvested in October, when fruit skin color changed from darker to lighter green and can be greenish yellow, and those free of blemishes and mechanical damage were collected [14].

The first sublots of fruits were used for transcriptome sequencing. The atemoya fruits were picked at about $80 \%$ ripening. Twenty fruits were kept at room temperature with no treatment, and recently picked fruits were defined as day 0 or NT-0. Most of the fruits without any treatment started to crack at the pedicel on day 2 and were named NT-PC-1, whereas splits increased and deepened on day 3 (NT-PC-2) and day 4 (NT-PC-2) with prolonged storage time. The following four stages were selected for transcriptome sequencing: recently picked fruits (NT-0), cracking at the pedicel (NT-PC-1), moderate cracking at the pedicel (NT-PC-2), and severe cracking at the pedicel (NT-PC-3). Another 20 fruits were immersed in $2 \mathrm{~g} / \mathrm{kg}$ ethephon for $2 \mathrm{~min}$, transferred into a closed plastic box, and the lid was left open after $24 \mathrm{~h}$. Fruits that did not exhibit cracking after $24 \mathrm{~h}$ of ethephon treatment were designated as Eth-24 h. Most fruits initially exhibited cracking in the pericarp on day 2 and were named Eth-PC. On day 3 , the fruits showed more extensive and deeper cracking, including the pericarp and the peduncle. However, not all of the fruits after ethephon treatment appeared cracking, and no-cracking fruits were defined as Eth-NC on day 4 after ethephon treatment. Samples were collected from three stages for transcriptome sequencing, including fruits $24 \mathrm{~h}$ after ethephon treatment (Eth-24 h), fruits with pericarp cracking after ethephon treatment (Eth-PC), and no fruit cracking after ethephon treatment (Eth-NC).

Three pericarp samples that showed similar TSS contents, firmness values, and degree of cracking under the same treatment at each stage were collected, immediately frozen in liquid nitrogen, and then stored at $-80^{\circ}$ $\mathrm{C}$ until further processing. Three replicate samples were prepared for each biological sample. The pericarps of the candidate samples, including NT-0, NT-PC-1, NT-PC-2, NT-PC-3, Eth-24h, Eth-PC, and Eth-NC, were subjected to transcriptome sequencing and expression analysis.

The second sublots of fruits were used for starch and pectin content determination. Fifteen fruits were stored at room temperature with no treatment, and another 15 fruits were treated with ethephon as earlier described. Samples were collected on days $0,1,3$, and 5. Three pericarp samples at each stage were collected, immediately 
frozen in liquid nitrogen, and then stored at $-80^{\circ} \mathrm{C}$ until further processing.

\section{Total soluble solids (TSS), firmness value, starch, and} pectin determination

TSS content and firmness were measured as previously described [22]. A solution of $80 \% \mathrm{Ca}\left(\mathrm{NO}_{3}\right)_{2}$ was used to extract starch from the ethanol-insoluble residue, and the absorption value of the reaction product at a wavelength of $620 \mathrm{~nm}$ was determined using the iodine-iodide kalium method according to the method of Cao et al. [50]. A solution of $95 \%$ ethanol was used to extract pectin in a boiling water bath. The supernatant was used to determine soluble pectin, and the ethanol-insoluble residue hydrolyzed by sulfuric acid was used to measure protopectin content according to the method Cao et al. [50]. Pectin was determined by carbazole, and the content of pectin was indicated by the quality of galacturonic acid.

\section{RNA extraction and illumina sequencing for transcriptome analysis}

Seven libraries (NT-0, NT-PC-1, NT-PC-2, NT-PC-3, Eth-24 h, Eth-PC, and Eth-NC) were designed for RNASeq to obtain a general overview of the atemoya pericarp transcriptome of fruit ripening and cracking. Total RNA was extracted from pericarp tissues using a Quick RNA isolation kit following the manufacturer's instructions (Huayueyang, China). The RNA samples were digested with DNase I to remove possible contaminating genomic DNA and purified with RNase-free columns (Huayueyang, China). RNA quality was verified using a 2100 Bioanalyzer RNA Nanochip (Agilent, Santa Clara, CA, USA), and RNA integrity was confirmed by electrophoresis on formaldehyde-containing $1.5 \%(w / v)$ agarose gels. Then, RNA was quantified using a NanoDrop ND-1000 spectrophotometer (Nano-Drop, Wilmington, DE, USA). Three high-quality RNAs were pooled by mixing equal quantities of RNA of the three biological replicates used in RNA-seq at each stage. Each library was sequenced in an Illumina HiSeq 2500 system using 100-bp paired-end protocols. All of the raw transcriptome data were deposited to the GenBank Short Read Archive (Accession number SRP114346).

\section{De novo assembly and functional annotation}

The resulting RNA sequencing data were subjected to purification, which included removing adapters and low-quality reads. Clean reads were de novo assembled using the Trinity Program [51]. The resulting sequences obtained with Trinity were then called unigenes. To annotate the unigenes, we used the BLASTx program (http:// www.ncbi.nlm.nih.gov/BLAST/) of NCBI with an E-value threshold of $1 \mathrm{e}-5$ to the NCBI non-redundant protein
(Nr) database (http://www.ncbi.nlm.nih.gov), the Universal Protein Resource (UniProt) database (http://www.uniprot.org/), the Kyoto Encyclopedia of Genes and Genomes (KEGG) database (http://www.genome.jp/kegg), the Gene Ontology (GO) database (http://geneontology.org/), and the Cluster of Orthologous Groups of protein (COG) database (http://www.ncbi.nlm.nih.gov/COG). Unigene expression level was normalized by calculating reads per kb per million reads (RPKM) [52]. Comparison of unigene expression among treatments was according to DEGSeq as described by Anders and Huber [53] and Wang et al. [54]. DEGs between treated and control samples were identified when the false discovery rate (FDR) $\leq 0.05$, $\mid \log 2$ ratio $\mid \geq 1$, and $P$-value $<0.05$. The library prepared from the NT-0 pooled samples was used as calibrators to normalize the DEGs in the other six libraries (NT-PC-1, NT-PC-2, NT-PC-3, Eth-24h, Eth-PC, and Eth-NC). DEGs were clustered by Short Time-series Expression Miner software (STEM) [55]. The clustered profiles with a P-value $\leq 0.05$ were considered to be significantly expressed. Then the DEGs were subjected to KEGG pathway annotation using Blast all software against the KEGG database. KEGG pathways with a Q-value $\leq 0.05$ are significantly enriched in DEGs. Senior bubble charts were constructed using the OmicShare tools, a free online platform for data analysis (www.omicshare.com/tools). To understand the gene expression patterns of DEGs during fruit cracking, we performed a hierarchical cluster analysis of expression patterns with cluster software [56] and Java TreeView [57].

\section{RT-qPCR validation}

RT-qPCR was applied to investigate gene expression patterns. First-strand cDNA was generated from $1 \mu \mathrm{g}$ total RNA isolated from the seven pericarp samples using the PrimeScript $^{\mathrm{TM}}$ RT reagent kit with gDNA Eraser (TaKaRa, Japan). RT-qPCR primers for were designed using Primer Premier 5.0 software (Premier, Canada) and synthesized by Sangon Biotech (Shanghai, China) Co., Ltd. The atemoya homologue actin (GenBank Accession Number MF893339) was selected as reference. All of the primers of the candidate unigenes are shown in Additional file 7. Each reaction (final volume: $10 \mu \mathrm{L}$ ) contained $5 \mu \mathrm{L}$ of $2 \times$ SYBR Green mastermix (Thermo Fisher), $1 \mu \mathrm{L}$ of each of the forward and reverse primers $(0.25 \mathrm{mM}), 1 \mu \mathrm{L}$ of the cDNA template, and $2 \mu \mathrm{L}$ of RNase-free water. RT-qPCR was conducted in a LightCycler 480 system (Roche, USA) using the following conditions: $95^{\circ} \mathrm{C}$ for $5 \mathrm{~min}$, followed by 40 cycles of $95^{\circ} \mathrm{C}$ for $30 \mathrm{~s}, 55^{\circ} \mathrm{C}$ for $30 \mathrm{~s}$, and $72{ }^{\circ} \mathrm{C}$ for $30 \mathrm{~s}$ in 96-well optical reaction plates. Each RT-qPCR analysis was performed in triplicate. Expression levels of the tested reference genes were determined by $\mathrm{CT}$ values and calculated using $2^{-\Delta \Delta \mathrm{Ct}}[58]$. 


\section{Statistical analysis}

All of the measurements were repeated thrice. All of the data were subjected to ANOVA according to the model for a completely randomized design using SPSS software (SPSS, Inc., Chicago, IL, USA). Differences between two sample evaluated by $t$ test at the 0.05 level in starch and pectin determination and differences among more samples were evaluated by Duncan's test at the 0.05 level in RT-qPCR analysis.

\section{Additional files}

Additional file 1: Figure S1. Size distributions of unigenes in the reference library. (DOC 21 kb)

Additional file 2: Figure S2. GO assignment of all of the unigenes in the reference Library. (DOC $195 \mathrm{~kb}$ )

Additional file 3: Figure S3. COG assignment of all of the unigenes in the reference library. (DOC $55 \mathrm{~kb}$ )

Additional file 4: Table S1. Pathway annotation of unigenes and the number of pathway from cracking atemoya pericarp. (XLSX $162 \mathrm{~kb}$ )

Additional file 5: Table S2. RPKM of the all of the unigenes in cracking pericarp of atemoya. (XLS $11116 \mathrm{~kb}$ )

Additional file 6: Figure S4. Profiles ordered according to $P$-value significance of the number assigned versus expected. Numbers between brackets indicate the number of the DEGs assigned. (DOC 2023 kb)

Additional file 7: Table S3. Primer sequences of the reference gene and candidate unigenes used in RT-qPCR analysis. (XLS $64 \mathrm{~kb}$ )

Additional file 8: Figure S5. Enriched starch and sucrose metabolism pathway. The components marked with red rectangles were considered differentially expressed. (DOC $32 \mathrm{~kb}$ )

\begin{abstract}
Abbreviations
1-MCP: 1-methylcyclopropene; AMY: Alpha-amylase; AMYG: Glucoamylase; BAM: Beta-amylase; bglB: Beta-glucosidase; bgIX: Beta-glucosidase; COI1: Coronatine-insensitive protein 1; CTR1: Serine/threonine-protein kinase; DBE: Starch debranching enzyme; DEGs: Differentially expressed genes; EIN2: Ethylene-insensitive protein 2; EIN3: Ethylene-insensitive protein 3; EXP: Expansins; GBSS: Granule-bound starch synthase; GID1: Gibberellin receptor GID1; glgA: Starch synthase; glgC: Glucose-1-phosphate adenylyltransferase; glgP: Starch phosphorylase; glgX: Glycogen debranching enzyme; HK: Hexokinase; JAZ: Jasmonate ZIM domain-containing protein; malQ: 4-alphaglucanotransferase genes; otsB: Trehalose 6-phosphate phosphatase; PE: Pectinesterase; PEL: Pectate lyase; PG: Polygalacturonase; PP2C: Protein phosphatase 2C; PUL: Pullulanase; PYL: Abscisic acid receptor PYR/PYL family; RTqPCR: Reverse transcription-quantitative real time PCR; SBE: Starch branching enzyme; scrK: Fructokinase; SS: Starch synthase; TPS: Trehalose 6-phosphate synthase/phosphatase; TREH: Alpha, alpha-trehalase; XET: Xyloglucan endotransglycosylase; YihQ: Alpha-glucosidase; AHK2_3_4: Arabidopsis histidine kinase 2/3/4; GAUT: Alpha-1,4- galacturonosyltransferase; glgB: 1,4-alpha-glucan branching enzyme
\end{abstract}

\section{Acknowledgements}

We thank LetPub (www.letpub.com) for its linguistic assistance during the preparation of this manuscript.

\section{Funding}

The project was supported by Central Public-interest Scientific Institution Basal Research Fund for Chinese Academy of Tropical Agricultural Sciences (No.1630062016003). The supporters had no role in study design, data collection, data analysis, data interpretation, the writing of the manuscript or decision to publish.

\section{Availability of data and materials}

All data supporting the findings is contained in the manuscript and its supplementary files except RNA-seq data. We have deposited our RNA-seq data in Sequence Read Archive (SRA) (http://www.ncbi.nlm.nih.gov/sra/), the accessions for our submission are SRP114346. The raw data for the DGE analysis were also deposited in the NCBI Sequence Read Archive under accession numbers SRX3170370 (NT-0), SRX3170369 (NT-PC-1), SRX3170368 (NT-PC-2) SRX3170367 (NT-PC-3), SRX3170372(Eth-PC), SRX3170373(Eth-24 h) and SRX3170371(Eth-NC) (http://www.ncbi.nlm.nih.gov/sra/).

\section{Authors' contributions}

CJJ performed most of the experiments and data analysis. DYJ and HUL carried out part of material collection, RNA extraction and physiology parameters determination. LWM conducted bioinformatics analyses and data interpretation. SDQ participated in the preparation of the manuscript. HHG and XJH conceived, designed and coordinated the studies. All authors have read and approved the final manuscript.

\section{Authors' information}

All authors works/study in the South Subtropical Crops Research Institute, Chinese Academy of Tropical Agricultural Sciences, Zhanjiang, 524091, China.

Ethics approval and consent to participate

Not applicable.

\section{Consent for publication}

Not applicable

\section{Competing interests}

The authors declare that they have no competing interests.

\section{Publisher's Note}

Springer Nature remains neutral with regard to jurisdictional claims in published maps and institutional affiliations.

\section{Author details}

${ }^{1}$ Key Laboratory of Tropical Fruit Biology, Ministry of Agriculture, South Subtropical Crops Research Institute, Chinese Academy of Tropical Agricultural Sciences, Zhanjiang 524091, China. ${ }^{2}$ National Field Genebank for Tropical Fruit (Zhanjiang), South Subtropical Crops Research Institute, Chinese Academy of Tropical Agricultural Sciences, Zhanjiang 524091, China.

Received: 13 October 2017 Accepted: 3 April 2019

Published online: 27 May 2019

\section{References}

1. Yamashita F, Migilioranza LHDS, Miranda LDA, Souza CMDAE. Effects of packaging and temperature on postharvest of atemoya. Rev Bras Frutic. 2002;24:658-60.

2. Pareek S, Yahia EM, Pareek OP, Kaushik RA. Postharvest physiology and technology of Annona fruits. Food Res Int. 2011;44:1741-51.

3. Beyer M, Hahn R, Peschel S, Harz M, Knoche M. Analysing fruit shape in sweet cherry (Prunus avium L.). Sci Hortic. 2002;96:139-50.

4. Sekse L, Bjerke KL, Vangdal E. Fruit cracking in sweet cherries-an integrated approach. Acta Hortic. 2005;667:471-4.

5. Simon G. Review on rain induced fruit cracking of sweet cherries (Prunus avium L.), its causes and the possibilities of prevention. Int J Hortic Sci. 2006; 12(3):27-35.

6. Hovland K, Sekse L. Water uptake through sweet cherry (Prunus avium L. )fruit pedicels: influence of fruit surface water status and intact fruit skin. Acta Agric Scand Sect B-soil Plant Sci. 2004;54:91-6.

7. Considine J. Physical aspects of fruit growth: cuticular fracture and fracture patterns in relation to fruit strue in Vitis vinifera. J Hortic Sci. 1982;57:79-91.

8. Khadivi-Khub. Physiological and gecturnetic factors influencing fruit cracking. Acta Physiol Plant. 2015;37:1718.

9. Li CR, Shen WB, Lu WJ, Jiang YM, Xie JH, Chen JY. 1-MCP delayed softening and affected expression of XET and EXP genes in harvested cherimoya fruit. Postharvest Biol Tech. 2009;52:254-9.

10. Liu KD, Liu JX, Li HL, Yuan CC, Zhong JD, Chen Y. Influence of postharvest citric acid and chitosan coating treatment on ripening attributes and 
expression of cell wall related genes in cherimoya (Annona cherimola mill.) fruit. Sci Hortic. 2016;198:1-11.

11. Mo YW, Gong DQ, Liang GB, Han RH, Xie JH, Li WC. Enhanced preservation effects of sugar apple fruits by salicylic acid treatment during post-harvest storage. J Sci Food Agr. 2008;88:693-2699.

12. Brown BI, Wong LS, George AP, Nissen RJ. Comparative studies on the postharvest physiology of fruit from different species of Annona (custard apple). J Hortic Sci. 1988;63:521-8.

13. Morena J, De La Plaza JL. The respiratory intensity of cherimoya during refrigerated storage: a special case of climacteric fruit? Acta Hortic. 1993;138: 179-86.

14. Paull RE. Postharvest atemoya fruit splitting during ripening. Postharvest Biol Tech. 1996:8:329-34

15. Rose JKC, Cosgrove DJ, Albersheim P, Darvill AG, Bennett AB. Detection of expansin proteins and activity during tomato fruit ontogeny. Plant Physiol. 2000;123:1583-92.

16. Giovannoni JJ. Molecular biology of fruit maturation and ripening. Annu Rev Plant Physiol Plant Mol Biol. 2001;52:725-49.

17. Wang Y, Lu WJ, Liu JG, Jiang YM. Differential expression of two expansin genes in developing fruit of cracking-susceptible and resistant litchi cultivars. J Am Soc Hortic Sci. 2006:131:118-21.

18. Kasai S, Hayama H, Kashimura Y, Kudo S, Osanai Y. Relationship between fruit cracking and expression of the expansin gene MdEXPA3 in 'Fuji' apples (Malus domestica Borkh.). Sci Hortic. 2008;116:194-8.

19. Shen WB, Li CR, Chen JY, Xie JH, Lu WJ. Expansin gene expression in cherimoya fruit is correlated with flesh firmness during fruit ripening and softening. J Hortic Sci Biotechnol. 2009;84:333-9.

20. Lu PL, Lin CH. Physiology of fruit cracking in wax apple (Syzygium samarangense). Botanica Orientalis J Plant Sci. 2011:8:70-6.

21. Li M, Zhang YM, Zhang ZY, Ji XH, Zhang R, Liu DL, et al. Hypersensitive ethylene signaling and ZMdPG1 expression lead to fruit softening and dehiscence. PLoS One. 2013;8:e58745.

22. Chen JJ, Duan YJ, Mo YW, Hu YL, Hu HG, Xie JH. Gene expression analysis related to cell wall metabolism in pericarp between custard apple cultivars susceptible and resistant to fruit cracking. J Fruit Sci. 2015;32:769-76

23. Balbontín C, Ayala H, Rubilar J, Cote J, Figueroa CR. Transcriptional analysis of cell wall and cuticle related genes during fruit development of two sweet cherry cultivars with contrasting levels of cracking tolerance. Chil J Agr Res. 2014;74:162-9.

24. Zhang FP, Chen WH, Lin DX. Technical specifications for post-harvest treatment of annona atemoya. Guangdong Agr Sci. 2003;3:43-5.

25. Li W, Chen WX. Advances in the studies on postharvest physiology and fresh-keeping methods of Annona fruit. J Trop Subtrop Bot. 2004;12(3):280-4.

26. Li WC, Wu JY, Zhang HN, Shi SY, Liu LL, Shu B, et al. De novo assembly and characterization of pericarp transcriptome and identification of candidate genes in response to fruit cracking in Litchi chinensis Sonn. Int J Mol Sci. 2014;15(10):17667-85.

27. Kong YZ, Zhou GK, Yin YB, Xu Y, Pattathil S, Hahn MG. Molecular analysis of a family of Arabidopsis genes related to galacturonosyltransferases. Plant Physiol. 2011;155(4):1791-805.

28. Gutiérrez M, Sola MM, Pascual L, Vargas AM. Postharvest changes of sugar concentration in chilled injured cherimoya (Annona cherimola mill.). J Plant Physiol. 1994;143:27-32.

29. Gutiérrez M, Sola MM, Pascual L, Rodriguez-Garcia MI, Vargas AM. Ultrastructural changes in fruit injured by chilling. Food Struct. 1992;11: 323-32.

30. Goñi O, Muñoz M, Ruiz-Cabello J, Escribano Ml, Merodio C. Changes in water status of cherimoya fruit during ripening. Postharvest Biol Tech. 2007;45:147-50.

31. Wills RBH, Poi A, Greenfield H, Righey CJ. Postharvest changes in fruit composition of Annona atemoya during ripening and effects of storage temperatures on ripening. HortScience. 1984;19:96-7.

32. Considine JA, Kriedemann PE. Fruit splitting in grapes: determination of the critical turgor pressure. Aust J Agric Res. 2000;23:7-24.

33. Verner $L$. Procedure for determining resistance of sweet cherry varieties to fruit cracking. Fruit Var Hortic Dig. 1957;12:3-4.

34. Huang XM, Li JG, Wang HC, Gao FF. The relationship between fruit cracking and calcium in litchi pericarp. Acta Hortic. 2001;558:209-15.

35. Gutiérrez M, Lahoz JM, Sola M, Pascual L, Vargas AM. Postharvest changes in total soluble solids and tissue $\mathrm{pH}$ of cherimoya fruit stored at chilling and non-chilling temperatures. Hortic Sci. 1994;69:459-63.
36. Jomngam P, Chen KY, Chumpookam J, Shiesh CC. Effect of ripening and storage temperatures on quality of atemoya (Annona cherimola mill. $\times A$. squamosa L.) fruit. Acta Hortic. 2017;1166:161-6.

37. Li WM, Chen JJ, Duan YJ, Hu HG, Pang ZC, Xie JH. Effect of the regulation of different temperature and ethylene treatments on polysaccharide metabolism during African pride winter fruits ripening and softening. Chin J Trop Crops. 2018;39(3):275-80.

38. Paull RE, Deputy J, Chen NJ. Changes in organic acids, sugars, and headspace volatiles during fruit ripening of soursop (Annona muricata L.). J Am Soc Hortic Sci. 1983;108:931-4.

39. Paull RE. Postharvest variation in composition of soursop (Annona muricata L.) fruit in relation to respiration and ethylene production. J Am Soc Hortic Sci. 1982;107:582-5.

40. Sola MDM, Gutiérrez M, Vargas AM. Regulation of hexose-phosphate cycle determines glucose and fructose accumulation in cherimoya (Annona cherimola mill.) during ripening. J Plant Physiol. 1994;144:569-75.

41. Schluepmann H, Dijken A, Aghdasi M, Wobbes B, Paul M, Smeekens S Trehalose mediated growth inhibition of Arabidopsis seedlings is due to trehalose-6-phosphate accumulation. Plant Physiol. 2004;135:879-90.

42. Dijken AJ, Schluepmann H, Smeekens SC. Arabidopsis trehalose-6phosphate synthase 1 is essential for normal vegetative growth and transition to flowering. Plant Physiol. 2004;135:969-77.

43. Devirgilio C, Hottiger T, Dominguez J, Boller T, Wiemken A. The role of trehalose synthesis for the acquisition of thermotolerance in yeast I genetic evidence that trehalose is a thermoprotectant. Eur J Biochem. 1994;219:179-86.

44. Mackenzie KF, Singh KK, Brown AD. Water stress plating hypersensitivity of yeasts: protective role of trehalose in Saccharomyces cerevisie. J Gen Microbiol. 1988;134:1661-6.

45. Hoda A, Khalil SHA. Cracking and fruit quality of pomegranate (Punica granatum L.) as affected by pre-harvest sprays of some growth regulators and mineral nutrients. J Hortic Sci Ornam Plants. 2013;5:71-6.

46. Cline JA, Trought M. Effect of gibberellic acid on fruit cracking and quality of Bing and Sam sweet cherries. Can J Plant Sci. 2007:1:545-52

47. Munish A, Kahlon PS, Mahajan BVC. Effect of exogenous application of growth regulators on fruit drop, cracking and quality of litchi (Litchi chinensis sonn.) CV. Dehradun. Agric Sci Digest. 2003;23:191-4.

48. Yilmaz C, Ozguven Al. Hormon physiology of preharvest fruit acking in pomegranate (Punica granatum). Acta Hortic. 2003;5:716-27.

49. Sharma SB, Dhillon BS. Endogenous level of gibberellins in relation to fruit cracking in litchi (Litchi chinensis Sonn.). J Res Punjab Agric Univ. 1986;23:432-4.

50. Cau JK, Jiang WB, Zhao YM. The experiment guide of fruits and vegetables postharvest physiology and biochemisty. 1rd ed. Beijing: Chinese Light Industry Press; 2007.

51. Grabherr MG, Haas BJ, Yassour M, Levin JZ, Thompson DA, Amit I, et al. Fulllength transcriptome assembly from RNA-Seq data without a reference genome. Nat Biotechnol. 2011;29:644-52.

52. Mortazavi A, Williams BA, McCue K, Schaeffer L, Wold B. Mapping and quantifying mammalian transcriptomes by RNA-Seq. Nat Methods. 2008:5:621-8.

53. Anders $\mathrm{S}$, Huber W. Differential expression analysis for sequence count data. Genome Biol. 2010;11:R106.

54. Wang LK, Feng ZX, Wang X, Wang XW, Zhang XG. DEGseq: an R package for identifying differentially expressed genes from RNA-seq data. Bioinformatics. 2010;26:136-8.

55. Ernst J, Bar-Joseph Z. STEM: a tool for the analysis of short time series gene expression data. BMC Bioinformatics. 2006;7:191.

56. de Hoon MJL, Imoto S, Nolan J, Miyano S. Open source clustering software. Bioinformatics. 2004:20:1453-4.

57. Saldanha AJ. Java Treeview-extensible visualization of microarray data. Bioinformatics. 2004:20:3246-8.

58. Livak KJ, Schmittgen TD. Analysis of relative gene expression Datausing realtime quantitative PCR and the $2^{-\triangle \Delta C t}$ method. Methods. 2001;25:402-8. 